\title{
Classification of Mammograms Using a Modular Neural Network
}

Timothy Cooley, Major, USAF, $\mathrm{PhD}$ and Evangelia Micheli-Tzanakou, $\mathrm{PhD}$

\author{
Dept of Biomedical Engineering, 'RUTGERS University \\ PO. BOX 909, Piscataway NJ 08855-0909 \\ e-mail:etzanako@rci.rutgers.edu
}

\begin{abstract}
The number of deaths linked to breast cancer has risen to 44,000 women annually. Early detection and treatment is the key to long term survival. Mammography remains the number one method for detection of breast cancer. Studies have shown that radiologists may differ in their interpretation of mammograms. A system is described which uses a digitized image of a mammogram and classifies it as normal or abnormal without the aid of an expert. This computer system processes the given image using multiresolution wavelet analysis and then extracts features using invariant moments. The extracted features are then used as inputs to a modular neural network that classifies the mammogram. Training results of 93.9 percent and validation results of 100 percent were achieved on a small data set.
\end{abstract}

Key words: Mammograms, multiresolution analysis, modular neural networks, classification.

\section{Introduction}

Mammography has been and still remains the key screening tool for breast abnormalities. Other modalities such as thermography, ultrasound, and magnetic resonance imaging are available, but none compares with the 
ease and low cost of mammography [Isard, 1984]. However, mammography is not without its problems [Davis, 1994]. Mammograms miss approximately 10 percent of all breast cancers. Even with recent advances it is as much an art as it is a science. Errors may occur at many stages starting with the very idea itself, then in the technique of the mammographer, and then to the radiologist who reads the mammograms. Mammograms are, simply, specialized x-rays [Dodd, 1984] and as such an abnormality will only appear if its absorption of the $\mathrm{x}$-ray waves is different from the surrounding tissue [Cowley and Ramo, 1993]. In some cases potential tumors are masked, because they appear strikingly similar to dense fibrous normal breast tissue [Davis, 1994]. Additionally, films from mammograms are notoriously difficult to read [Cowley and Ramo, 1993; Vyborny and Giger 1994], as they are low in contrast and high in noise. Because of these factors it is no wonder that a recent report showed that consistency among radiologists' interpretation of mammograms was moderate, and in several cases the readings differed substantially [Elmore et al., 1994].

In the last few years, a lot of research has been done on mammography, using different methods of processing and interpretation [Dhawan et al., $1986,1988,1993]$. Given the difficulty in reading mammograms and the benefit of early detection of breast cancers, it is not surprising that much research has been done in recent years on computerized systems to aid in this process. This research has focused on two major areas: developing a system that enhances mammograms and makes them easier for a radiologist to read, and systems that take regions of interest selected by a radiologist to determine whether these regions are malignant or benign. The first area falls under the category of Computer Aided Diagnosis (CAD) systems and centers on helping detect possible cancer areas. The second area focuses on reducing the number of biopsies performed each year, and still requires the radiologist to perform the first step of the detection. In both cases, the expert (radiologist) must be part of the system.

A different approach to this problem would be to develop a system which reads the mammogram by using the entire image and then gives a classification of that mammogram. A radiologist will classify a 
mammogram into one of five categories: Category I is completely normal; Category II shows a known abnormality that has appeared previously and is benign; Category III shows an abnormality that is thought to be benign--a follow-up sooner than normal may be recommended but a biopsy should not be done; Category IV shows a suspicious abnormality that needs further evaluation and probably biopsy; and Category $\mathrm{V}$ shows a definite cancerous tumor that requires immediate attention. A computer system that could perform this type of classification could essentially act as a second opinion for a radiologist. One important benefit is that the computer system would be consistent and the inconsistencies noted by Elmore et al. [Elmore et al., 1994] would no longer be a problem. However, even if the system could just delineate normal mammograms (Category I) from abnormal ones (Categories II, III, IV and V) this would be of help. In this case, the system could still be used as a second opinion and might detect some mammograms that are classified as normal that are really abnormal. Additionally, there is some variation between radiologists over what is a category III mammogram and what is a category IV. The difference between these two categories is somewhat subjective. There are some radiologists who choose the cautious side and recommend a biopsy on anything that is a change. Others will hold offion the biopsy but recommend a follow-up in three to six months. Because of this, any system developed to classify mammograms in all five categories would be biased toward the radiologist reading the mammograms.'

Another area where a system that could delineate between normal and abnormal would be of use is in places where there is no radiologist. One of the most prominent places where this occurs is the mobile mammography van. These vans travel primarily to major corporations and offer women an opportunity to have a mammogram done with minimal disruption to their routine. All they need to do is essentially schedule approximately 30 minutes for the procedure and then they are back at work. This is much more

\footnotetext{
' This system was designed when only four categories were used. Previously category I included those mammograms that were totally normal as well as those that had a known abnormality. Five categories were created when category I was split as defined above.
} 
appealing and convenient than driving to a center and having to schedule perhaps a couple of hours for travel time and the appointment. However, in the mobile van once the mammograms are taken they are not read until the following day. This means that if there is a need for further views or perhaps for some reason the $\mathrm{x}$-ray is unreadable, the woman must now schedule a follow-up appointment at a center. If the mammography technician knew at the time of the first exam whether the mammogram was normal or abnormal, the extra views could be taken right then.

This paper discusses the design, methods and results of a computer system that classifies mammograms as normal or abnormal. The motivation for this kind of research has been given above, but succinctly stated the system is intended to aid in the early detection of breast cancer. The goal of this research is to develop a system which can take a mammogram and classify it as normal or abnormal with no expert intervention. Additionally, the system should be allowed to be mobile in the sense that it could be fielded on a mobile mammography van. For this system to function in that capacity, it must be able to produce a reading quickly enough so that the wait time for the patient is not unacceptable. Eventually digital mammography will be the standard, but until that time this system must have the capability to digitize the mammograms as well.

\section{Methods and System Overview}

The system described in this paper is comprised of three essential steps divided into two developed programs. The three basic elements are: Image acquisition, Image transformation and preprocessing in order to extract features for classification, and the classification itself via neural networks. Acquiring, digitizing or scanning the image is a step which requires the use of a commercial scanning system, one that can accurately scan transparencies or $\mathrm{x}$-ray films at a high resolution. The transforming and processing module and the classification process are developed software and 
will comprise the major part of the discussion of the next two sections. Figure 1 shows a block diagram of the system.

\subsection{Data Acquisition}

As previously stated, digitizing a mammogram image can be accomplished by using one of several good scanning systems commercially available. Still, there are some issues which necessitate discussion.

A radiologist reads a mammogram looking for submillimeter microcalcifications as well as larger nodules or tumors [Sickles, 1986]. Because of this, any system that is used to scan these images must have a high enough resolution to capture these small microcalcifications. Additionally, the scanner must be able to scan $x$-ray images so that they appear to have some depth and not just as a flat two-dimensional image. By using a scanner with a transparency adapter, images were obtained which more closely resembled the true mammogram film. Our scanner had a resolution of 400 dots $/ \mathrm{inch}$, corresponding to $.0625 \mathrm{~mm} / \mathrm{pixel}$ (or 62.5 microns $[\mu \mathrm{m}])$, which is adequate to capture the small microcalcifications. Additionally, the scanning system had software which allowed the contrast and brightness values to be adjusted. By using this software the images were essentially preprocessed by enhancing the contrast and obtaining the best possible image. While this does not reduce the noise in the image it is a

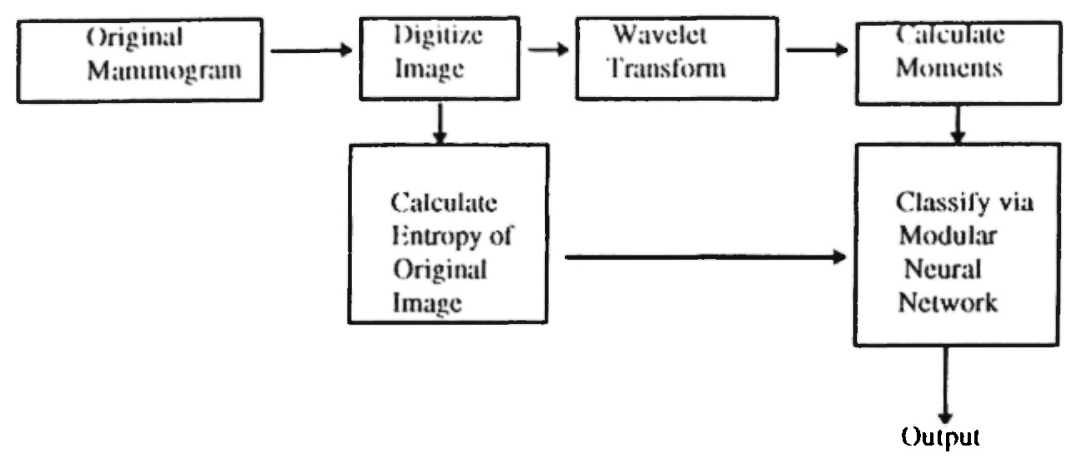

Fig. 1: Overview of the System 
good first preprocessing step. This resulted in files that were 3 megabytes to 9 megabytes in size. The sizes vary since mammograms vary in size, and to save space each image was windowed so that the absolute minimum area was captured. This scanning system saved the files in Microsoft Windows 3.x bitmap (BMP) format and therefore, all processing assumes this format.

\subsection{Feature Extraction by Transformation}

Since the raw digitized images are very large, some method of reducing the number of data points or extracting features must be applied prior to attempting to classify the images. Additionally, mammogram images are known to be noisy [Vyborny and Giger, 1994]. However, there are conflicting opinions as to whether it is desirable to filter the image [Bankman et al., 1993; Qian et al., 1994]. On the one hand, it is difficult to distinguish noise from the microcalcifications, and any filtering may result in essential data loss. The other opinion is that the noise appears too much like the real microcalcifications and must be eliminated in order to reduce the number of false-positive readings that are obtained. The ideal situation would be to filter out the noise only and leave all the microcalcifications intact. While the perfect ideal filter may not exist, a multiresolution technique solves some of the problems.

The wavelet transformation with multiresolution analysis was chosen for the first stage of the processing. Not only does the wavelet transform tend to extract the critical areas, but the multiresolution method performs an essential task. Wavelets and multiresolution analysis together have recently been widely used. For example, they have been used: to detect microcalcifications in mammograms [Laine et al., 1994; Strickland and Hahn 1996; Qian et al., 1994; Yoshida et al., 1995], to extract features in face recognition [Micheli-Tzanakou et al., 1995], to compress images [Goldberg et al., 1994; Kim et al., 1996; Zettler et al,. 1990], and to enhance images and simulate human perception [Myers et al., 1995].

Mammograms are difficult images as they must be viewed at many different scales [ $D^{\prime}$ Orsi and Kopans, 1993]. At a coarse scale, a relatively 
large tumor may be found which would cause a mammogram to be abnormal. These are the obvious and easier classifications. The microcalcifications appear at a fine scale, which if found in clusters will also result in an abnormal mammogram. These microcalcifications are extremely difficult to detect [Sickles, 1986; Vyborny and Giger, 1994] and virtually impossible to find if searched for on a coarse scale. Multiresolution analysis allows the image to be analyzed at a fine scale first and then successively coarser scales at each level [Mallat, 1989]. Additionally, wavelet functions have the ability to be translated and dilated so that in the wavelet analysis small transient signals are able to be detected [Daubechies, 1992; Sheng, 1996]. This ability is what enables them to extract the small microcalcifications as the microcalcifications appear in an image, in much the same way a transient spike appears in a signal. Furthermore, the wavelet transform is a fast transformation. It is implemented via convolutions which makes the complexity linear in the size of the input $(\mathrm{O}(\mathrm{n}))$ [Cody, 1992; Strang, 1994]. While this is still a lengthy process on large images, it is the best transformation complexity that can currently be achieved. Multiresolution and wavelet transformations are discussed below.

While the wavelet transform highlights the potential problem areas, it does not reduce the number of data points. On the contrary, since the images were transformed to five octaves the result was 33 percent more data points. Therefore, a feature extraction method was needed. Recent success with classifying images by using invariant moments [Micheli-Tzanakou et al., 1995] led to this direction. Mammography is not immune to some of the standard problems of imaging, i.e. translation, rotation, and scaling. It is easy to see that from one mammogram to the next, even on the same patient, it is highly unlikely that the breast will be positioned in exactly the same place on the $x$-ray film. Additionally, rotation is a problem since the procedure itself does not always take the mammogram from the same angle. Even without the variance in the procedure it would be unlikely that the breast would not be rotated on the film. In order to obtain a better image of the tissue the breast is compressed in the mammogram process. Since this is a subjective process the amount of compression may vary slightly from one 
mammogram technician to another. This, of course, changes the shape or the scaling of the image. Additionally, a patient may gain or lose weight between two mammogram procedures (usually there is a year or more between mammogram appointments). This also may affect breast size. Because of all these variables, moments which are invariant to rotation, translation and scaling appeared to be a logical choice as features for the classification process. The $\mathrm{Hu}$ moments [Hu, 1962] met these qualifications and were chosen. However, the moments were calculated not only on the original image, but also on the transformed components. This is important as the wavelet multiresolution analysis has reduced the noise and highlighted the potential problem areas. In Shen et al. [1994] it is noted that moments are susceptible to noise and may not perform well on noisy images. The wavelet transform has reduced the noise in most cases and increased the performance of the moments as features. Moments are discussed in more detail below.

Since there are seven Hu invariant moments through the third order, the number of data points was reduced at this step to 147 (i.e five octaves of transforms with 4 components per octave equals 20 components. Seven moments of 20 components equals 140 moments, plus the moments of the original image equals 147). In viewing the wavelet transforms it was observed that the fifth octave did not contain much information (due to most of the details being extracted at this point) and, therefore, was not used in the classification process. This results in only 119 data points.

In the initial design the moments were not calculated on the original image. Because of this it was determined that some measure from the original image might aid in the classification process. One such compact and global measure is the entropy of the image. Widely used in information theory as a measure of the average number of bits required to represent each symbol in an alphabet [Held, 1987], it also can be a measure of the information content of a signal or image [Ross, 1976].

With the addition of the entropy of the image there are 120 data points per image used in the classification process. This represents a huge reduction in dimensionality which is what was needed. The average 
mammogram image digitizes to 4.5 megabytes. Representing the features in 120 data points means the dimensionality has been reduced on the order of $10^{5}$.

\subsubsection{Multiresolution Analysis}

Recent years have seen a dramatic increase in the study of wavelet functions and wavelet transformations [Richardson, 1995]. Since 1989, when Mallat [Mallat, 1989] linked wavelet functions and multiresolution analysis, it appears that the two have mistakenly been forever blended. Multiresolution analysis is a very useful technique and can stand on its own without wavelet functions. The Laplacian pyramid and subband coding are two examples of multiresolution analysis techniques that do not require the use of wavelets. On the other hand, wavelet functions and transformations exist as mathematical entities wholly apart from multiresolution analysis. The two of them together provide a powerful tool. Multiresolution analysis was first presented by Burt and Adelson [Burt and Adelson, 1983] in 1983 for image decomposition, coding and reconstruction. There are two main approaches to multiresolution analysis, the Laplacian pyramid and the subband coding technique [Sheng, 1996].

The basic idea of subband coding is to split the frequency band of a signal into subbands and then process or code each subband individually [Woods and O'Neil, 1986]. It has been used extensively in speech coding, whereby each subband is coded based upon the statistics of that band. This allows the coding error to be confined to a distinct band as well as shaping the noise spectrum by subband such that the apparent noise is minimized [Woods and O'Neil, 1986]. In addition, subband coding can be used in image compression algorithms, whereby better compression is obtained by adapting the compression to the individual subbands [Woods and O'Neil, 1986]. While both the Laplacian pyramid and subband coding involve a lowpass filtering process, the detail signals are obtained much differently. Obtaining the detail signal with a Laplacian pyramid involves upsampling the low-resolution signal. With the subband coding technique the detail signal is obtained by high-pass filtering [Daubechies, 1992; Sheng, 1996]. 
One benefit of this technique is that instead of the detail signal, $d_{i}(n)$, being the same length as $\mathbf{x}_{i}(n)$, the original signal, as in the Laplacian pyramid, the subband coding method produces a detail signal that is half the length [Sheng, 1996]. Therefore, the result from subband coding is a half-length, low-resolution signal and a half-length detail signal.

\subsubsection{Wavelets}

Wavelets are a family of functions that has been the topic of great study since the early 1980s. However, early research on these functions date back as far as 1964. One of the key properties of wavelets is that they are nonzero over a finite portion of their domain and, therefore, are good choices for the analysis of local phenomena in signals and images (images being considered as just a two-dimensional signal) [Daubechies, 1992]. Recent fame has been brought to these functions by Daubechies [Strang, 1994], but perhaps equally important to image processing research is the work done by Mallat [Mallat, 1989]. No matter who is credited with bringing wavelets to the forefront, they have now been used fairly extensively in many different types of signal and image processing applications.

Wavelets are defined by the family of functions:

$$
h_{s, \tau}(t)=\frac{1}{\sqrt{s}} h\left(\frac{t-\tau}{s}\right)
$$

where $s$ is the scaling factor and $\tau$ is the translation factor. The ability to change scale and position makes wavelets very powerful functions for signal analysis [Rioul and Vetterli, 1991]. (The one-dimensional case is presented for clarity. The extension to two dimensions can be readily made). Wavelets are generated from the standard wavelet function $h(t)$ by scaling and translating. When $s>1$, the functions are dilated and when $s<1$ the functions are contracted. The constant factor $\frac{1}{\sqrt{s}}$ is included in order to normalize the wavelets [Sheng 1996]. When the factor is $\frac{1}{\sqrt{s}}$, the wavelets are normalized with respect to energy, so that: 


$$
\int|h s, \tau(t)|^{2} d t=\int|h(t)|^{2} d t=1
$$

If the factor is $\frac{1}{\mathrm{~S}}$ then the wavelets are normalized with respect to amplitude and:

$$
\int|h(t)| d t=1
$$

Even when scaled and translated, all wavelets $h_{1 . \tau}(t)$ generated from the same wavelet $h(t)$ have the same identical shape [Sheng, 1996]. Figure 2 shows the Mexican hat-like wavelet:

$$
h(t)=(1-t) e^{\frac{-t^{2}}{2}}
$$

and then the same $h(t)$ dilated by 2 and translated by 3 .

Wavelets may also be used as basis functions [Antonini et al., 1992; Cody, 1992; Daubechies, 1992; Strang, 1994]. Consider a function $f(t)$. Then the continuous wavelet transform of $f(t)$ is:

$$
W_{f}(s, \tau)=\int f(t) h_{s . \tau}^{*}(t) d t
$$

where $h^{*}(t)$ is the complex conjugate of $h(t)$. In practice, however, virtually all wavelets are real-valued functions [Sheng, 1996]. Note that in the
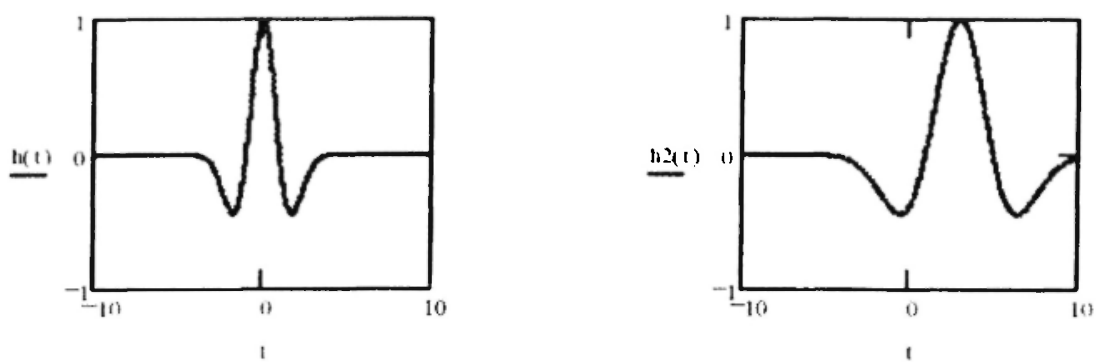

Fig. 2: Mexican Hat Wavelet -The left graph shows $h(t)$ undilated and centered on $t=0$. The right graph shows $h(t)$ dilated by a factor of 2 and translated +3 in the $t$ direction. 
definition of the wavelet transform the wavelet basis functions are not specified [Sheng, 1996]. This is in direct contrast to Fourier transform or any other transform , and is not dependent upon one set of basis functions. The theory of wavelets deals with the general properties of wavelets and the wavelet transforms and is not dependent upon one set of basis functions. Specific wavelet bases may be built to fit the need of a certain application. There are many kinds of wavelet bases [Antonini et al., 1992; Sheng, 1996]: orthogonal, orthonormal, continuous, discrete, nonorthogonal, etc. However, all wavelets must oscillate and have their mean value equal to zero [Rioul, and Vetterli, 1991; Sheng, 1996]. This is called the "admissibility condition." In addition, all wavelets must have exponential decay so that they are local in scope [Daubechies, 1992; Sheng, 1996]. This is called the "regularity condition." Therefore, in the time domain wavelets are just little waves that oscillate quickly and then vanish once outside a specified domain, hence their name [Sheng, 1996].

The wavelet transform of a function $f(t)$ transforms a function in the time domain to one in the time-frequency domain [Hlawatsch and BoudreauxBartels, 1992]. Thus a one-dimensional function becomes two-dimensional after the transform. One clear example of the time-frequency domain is the notation used in musical scores [Sheng, 1996; Strang, 1994]. At each point in time in the musical score a musician plays a note or frequency. The placement of a note on the horizontal axis of a musical staff tells the musician when to play the note, and the height of the note on the vertical axis determines what frequency (or note) to play. In this manner, musical scores are a time-frequency joint representation, the same as the domain of the wavelet transform. It is worth noting, however, that the resolution in the time-frequency plane is limited by the Heisenberg inequality [Hlawatsch and Boudreaux-Bartels, 1992; Rioul and Vetterli, 1991; Sheng, 1996] which states:

$$
\Delta \mathrm{t} \Delta \omega>\frac{1}{2}
$$

Therefore, a signal cannot be represented by a point in space but only by a rectangle of $\Delta t \Delta \omega$ in the time-frequency plane. This is also important as it 
is easy to see that if one desires a fine time resolution it will result in a coarse frequency resolution, and a fine frequency resolution results in a coarse time resolution.

To this point we have only discussed the continuous wavelets and wavelet transforms. Most of the time in actual use we utilize the discrete version, since a computer represents signals and images as discrete entities. While the mathematics of the transform involve sums and not integrals as in the continuous case, the theory applies equally to both. As the actual implementation of the wavelet transform is discussed, the discrete wavelet transform will be introduced.

\subsubsection{Wavelets and Multiresolution Analysis}

Subband coding is an orthonormal signal decomposition and reconstruction method. What was discovered was that orthonormal wavelet bases perform well when used in the subband coding technique [Mallat, 1989]. Because of their local nature, wavelets provide a good framework for multiresolution analysis of time-varying signals that may contain transient spikes [Daubechies, 1992].

When using wavelets with multiresolution analysis, a scaling function, $\phi(t)$, is introduced. The scaling function is normally continuous, can be dilated and translated and is generally real-valued [Sheng, 1996]. However, it is not a wavelet, as it does not need to satisfy the wavelet admissibility condition and have a zero mean [Sheng, 1996]. Scaling functions, however, are usually normalized such that:

$$
\int \phi(t) d t=1
$$

In the multiresolution framework the scaling function plays the role of the smoothing or averaging function [Sheng, 1996]. The scaling function with translation parameter $k$ and scaling factor $s$ is defined as:

$$
\phi_{i, k}(t)=\frac{1}{\sqrt{2^{i}}} \phi\left(\frac{t}{s^{i}}-k\right)
$$


This dilated $\phi_{i, k}(t)$ forms an orthonormal basis at each resolution level $i$ due to the discrete translations. When $s=2$, which is the usual case, the scaling is called dyadic [Sheng 1996]. Each dilated version of $\phi_{i k}(t)$ has the same basic shape as the original scaling function, $\phi(t)$. This is the same property as the dilated wavelets shown in Figure 2.

Since the $\phi(t)$ form an orthonormal bases, they must span a subspace. Call this subspace $V_{0}$. If $\phi(t)$ is then contracted by a factor of 2 and translated in half integer steps, the set $\{\phi(2 t-k)\}$ is formed. This set spans the next finer resolution subspace, $V_{-1}$ and $V_{0} \subset V_{-1}$ [Antonini et al., 1992; Sheng, 1996]. However, since the set $\{\phi(2 t-k)\}$ is an orthonormal basis in $V_{-1}$, then $\phi(t)$ may be written as a linear combination of the weighted sum of the functions in this set [Rioul and Vetterli, 1991]. Or,

$$
\phi(\mathrm{t})=\sum_{\mathbf{k}} \mathrm{p}(\mathrm{k}) \phi(2 \mathrm{t}-\mathrm{k})
$$

for $k \in Z$. This is called the two-scale relation or two-scale difference equation with $\mathrm{p}(\mathrm{k})$ called the "interscale coefficients" [Sheng, 1996]. Additionally, Strang [Strang, 1994; Strang, 1989] calls this equation the "dilation equation".

The wavelet functions, $\Psi(\mathrm{t})$, act as the high-pass filter in the subband coding scheme and may also be written as the sum of the scaling functions, since they belong to the same subspace [Rioul and Vetterli, 1991; Sheng, 1996]. Therefore,

$$
\Psi(\mathrm{t})=\sum_{\mathrm{k}} \mathrm{q}(\mathrm{k}) \phi(2 \mathrm{t}-\mathrm{k})
$$

Note that in the left-hand side of equations 9 and 10 the functions $\phi(t)$ and $\Psi(t)$ are continuous functions, but the right-hand sides of the equations are discrete. Thus the two-scale relation expresses the relationship between the continuous functions and the interscale coefficients [Sheng, 1996; Strang, 1994]. These interscale coefficients are the filter coefficients for the low- and high-pass filters used in the subband coding algorithm. 
While there are many ways to calculate the interscale coefficients, virtually every method starts with the two-scale relation in some form. However, because of the cross-orthogonality condition, only the low-pass coefficients need to be calculated [Cody, 1992; Sheng, 1996]. The high-pass coefficients may then be calculated from the low-pass by the relation:

$$
q(n)=(-1)^{n+1} p(N-1-n)
$$

where $\mathrm{N}$ is the filter length (must be even) and $\mathrm{n}=0,1,2 \ldots \mathrm{N}-1$

Daubechies [Daubechies, 1992] uses the regularity condition on wavelets in the frequency domain to calculate the filter coefficients. While this is more general, the mathematics are complex. Others use the two-scale relation in the frequency domain to determine the coefficients. Sheng [Sheng, 1996] gives examples of these techniques as well. Most often the desired filter coefficients can be found in a table in a journal article or in another reference. Daubechies [Daubechies, 1992] lists coefficients for several wavelets of all different sizes (size refers to the number of filter coefficients) in her book.

\subsubsection{Invariant Moments}

Moments have long been used in statistical theory and classical mechanics [Yu and $\mathrm{Li}, 1990$ ]. Statisticians view moments as means, variances, skewness, and kertosis [Levine, 1985] of distributions, while classical mechanics students use moments to find centers of mass and moments of inertia [Thomas and Finney, 1988]. In imaging, moments have been used as feature vectors for classification [Micheli-Tzanakou et al., 1995] as well as for image texture attributes [Levine, 1985] and shape descriptors of objects [Gonzalez and Wintz, 1977; Reeves et al., 1988; Shen et al., 1994]. In the early 1960s, Hu developed seven invariant moments from algebraic moment theory [Hu, 1962]. These seven moments are invariant under translation, rotation, and scaling. Perhaps the most important contribution of this work was the application of these seven invariant moments to the two-dimensional pattern recognition problem of 
character recognition. This was a crucial development, since many key problems in imaging and image recognition focus on recognizing an image even though it has been translated or rotated, or perhaps magnified by some means. Since that time other pattern recognition applications have included hand-printed characters, aircraft identification, chest $x$-rays and ship recognition [Levine, 1985], and more recently face recognition [MicheliTzanakou et al., 1995].

Similar to the definition of moments in classical mechanics, the twodimensional moments of order $(p+q)$ with an image intensity distribution of $f(x, y)$ are defined as:

$$
m_{p q}=\iint x^{p} y^{q} f(x, y) d x d y
$$

where $p, q=0,1,2, \ldots$ These moments in general are not invariant to any distortions [Yu and $\mathrm{Li}, 1990]$ and, therefore, the central moments are defined as:

$$
\mu_{p q}=\iint\left(x-x^{\prime}\right)^{p}\left(y-y^{\prime}\right)^{q} f(x, y) d\left(x-x^{\prime}\right) d\left(y-y^{\prime}\right)
$$

where $x^{\prime}=\frac{m_{10}}{m_{00}}$ and $y^{\prime}=\frac{m_{\hat{v}}}{m_{00}}$. The central moments are known to be invariant under translation, and by working through equation 13 it can be shown that the first four orders of central moments can be expressed in terms of the ordinary moments defined in equation 12 as:

$$
\begin{aligned}
& \mu_{00}=\mathrm{m}_{00} \\
& \mu_{10}=\mu_{01}=0 \\
& \mu_{11}=\mathrm{m}_{11}-\frac{\mathrm{m}_{10} \mathrm{~m}_{01}}{\mathrm{~m}_{00}} \\
& \mu_{20}=\mathrm{m}_{20}-\frac{\mathrm{m}_{10}^{2}}{\mathrm{~m}_{00}} \\
& \mu_{02}=\mathrm{m}_{02}-\frac{\mathrm{m}_{01}^{2}}{\mathrm{~m}_{00}}
\end{aligned}
$$




$$
\begin{aligned}
& \mu_{12}=m_{12}-m_{02} x^{\prime}-2 m_{11} y^{\prime}+2 m_{10} y^{\prime^{2}} \\
& \mu_{21}=m_{21}-m_{20} y^{\prime}-2 m_{11} x^{\prime}+2 m_{01} x^{\prime^{2}} \\
& \mu_{03}=m_{03}-3 m_{02} y^{\prime}+2 m_{01} y^{y^{2}} \\
& \mu_{30}=m_{30}-3 m_{20} x^{\prime}+2 m_{10} x^{\prime 2}
\end{aligned}
$$

Often it is desirable to normalize the moments with respect to size. This may be accomplished by using the area, $\mu_{00}$. The normalized central moments are:

$$
\eta_{\mathrm{pq}}=\frac{u_{\mathrm{pq}}}{u_{00}^{\gamma}}
$$

where $\gamma=1+\frac{(p+q)}{2}$ for $(p+q)=2,3, \ldots \ldots$

With these normalized moments the seven $\mathrm{Hu}$ invariants are found as given in [Gonzalez and Wintz 1977] by:

$$
\begin{aligned}
& \varphi_{1}=\eta_{20}+\eta_{02} \\
& \varphi_{2}=\left(\eta_{20}-\eta_{02}\right)^{2}+4 \eta_{11}{ }^{2} \\
& \varphi_{3}=\left(\eta_{30}-3 \eta_{12}\right)^{2}+\left(3 \eta_{21}-\eta_{03}\right)^{2} \\
& \varphi_{4}=\left(\eta_{30}+\eta_{12}\right)^{2}+\left(\eta_{21}+\eta_{03}\right)^{2} \\
& \varphi_{5}=\left(\eta_{30}-3 \eta_{12}\right)\left(\eta_{30}+\eta_{12}\right)\left[\left(\eta_{30}+\eta_{12}\right)^{2}-3\left(\eta_{\cdot 21}+\eta_{03}\right)^{2}\right] \\
& +\left(3 \eta_{21}-\eta_{03}\right)\left(\eta_{21}+\eta_{03}\right)\left[3\left(\eta_{30}+\eta_{12}\right)^{2}-\left(\eta_{21}+\eta_{03}\right)^{2}\right] \\
& \varphi_{6}=\left(\eta_{20}-\eta_{02}\right)\left[\left(\eta_{30}+\eta_{12}\right)^{2}-\left(\eta_{21}+\eta_{03}\right)^{2}\right] \\
& +4 \eta_{11}\left(\eta_{30}+\eta_{12}\right)\left(\eta_{21}+\eta_{03}\right) \\
& \varphi_{7}=\left(3 \eta_{21}-\eta_{03}\right)\left(\eta_{30}+\eta_{12}\right)\left[\left(\eta_{30}+\eta_{12}\right)^{2}-3\left(\eta_{21}+\eta_{03}\right)^{2}\right] \\
& +\left(3 \eta_{12}-\eta_{03}\right)\left(\eta_{21}+\eta_{03}\right)\left[3\left(\eta_{30}+\eta_{12}\right)^{2}-\left(\eta_{21}+\eta_{03}\right)^{2}\right]
\end{aligned}
$$

While these seven invariants are given in terms of the normalized moments, they may be calculated from the central moments as well [Levine, 
1985]. In that case the formulas are the same with $\mu$ substituted for $\eta$ in equation 16.

One item to note is that these normalized moments assume that the image is represented by pixels, the values of which are all $>0$. There is no problem in calculating the original and central moments even if some of the pixels are $<0$. However, the normalized moments pose a different problem. If a substantial number of pixels have values $<0$ then $\mu_{00}$ becomes negative. This causes a problem during the normalization, since $\mu_{00}$ raised to a nonintegral power becomes an imaginary number. Because this system calculates the moments of the wavelet coefficients there are situations where a substantial number of coefficients are negative. Because of this, when the normalized moments are calculated $\mu_{00}$ is treated somewhat like a vector. It is considered to have magnitude $\left|\mu_{00}\right|$ and a direction which is + if $\mu_{00}>0$ and - if $\mu_{00}<0$. Therefore, the normalization of $\mu_{p q}$ is then:

$$
\eta_{\mathrm{pq}}=\frac{u_{\mathrm{pq}}}{u_{00}^{\gamma}} \text { if } \mu_{00}>0 \quad \eta_{\mathrm{pq}}=-\frac{\mu_{\mathrm{pq}}}{\left|\mu_{00}\right|^{\gamma}} \text { if } \mu_{00}<0
$$

Therefore, this technique actually calculates pseudo-moments to use as features.

\subsubsection{Entropy}

Entropy is a quantity that is widely used in information theory and is based on probability theory [Held, 1987; Ross, 1976]. Consider first an event $E$ that can occur when an experiment is performed. How surprised would one be to see that $\mathrm{E}$ actually does occur? The answer to that question depends upon the probability of E. Suppose, for instance, cards are being drawn with replacement one at a time from a full deck of playing cards. If $\mathrm{E}$ were defined as "the card being a heart" it would not be too surprising if $\mathrm{E}$ occurred as $P(E)=0.25$. (There are 52 cards in a full deck broken into 4 equal suits, hearts, spades, diamonds, and clubs). However, if $E$ were defined to be "the card being the ace of hearts" then we might be rather surprised to see that occurring, as now $P(E)=0.0192$ or $\frac{1}{52}$, but it is possible to quantify this concept of surprise [Ross, 1976]. 
If this concept is extended to a random variable $\mathbf{X}$ which can be one of the values $x_{1}, x_{2}, \ldots, x_{n}$ with probability $p_{1}, p_{2}, \ldots, p_{n}$, then the expected amount of surprise upon learning the value of $\mathbf{X}$ is:

$$
H(\mathbf{X})=-\sum_{i=1}^{n} p_{i} \log _{2} p_{i}
$$

This quantity is the entropy of the random variable $\mathbf{X}$. Note that if $p_{i}=0$, then $0 \log _{2} 0$ is defined to be 0 .

Thus $H(\mathbf{X})$ represents the average amount of surprise associated with the value of $\mathbf{X}$. It also can be interpreted as representing the amount of uncertainty that exists in the value of $\mathbf{X}$. In information theory $H(X)$ is considered to be the average amount of information received when the value of $X$ is observed [Held, 1987].

It is from the information theory point of view that the entropy would be a valid data point for images. It was conjectured that perhaps normal mammograms would contain more information than abnormal or vice-versa, and therefore, the entropy values would be of use for classification purposes. Interestingly enough, when viewed by a human observer the distribution of entropy values of abnormal mammograms seemed no different than the distribution of the normal mammogram entropy values. However, when used as input to the neural network they indeed added some discrimination. This is further discussed in the results section.

\section{Modular Neural Networks}

Simon Haykin in his book Neural Networks - A Comprehensive Foundation [Haykin, 1994] defines a modular neural network as:

"A neural network is said to be modular if the computation performed by the network can be decomposed into two or more modules (subsystems) that operate on distinct inputs without communicating with each other. The outputs of the modules are mediated by an integrating unit that is 
not permitted to feed information back to the modules. In particular, the integrating unit both (1) decides how the outputs of the modules should be combined to form the final output of the system, and (2) decides which modules should learn which training patterns."

Figure 3 shows an example structure of a modular neural network.

The idea of modular neural networks is analogous to biological systems [Haykin, 1994; Hrycej, 1992]. Our brain has many different subsystems that process sensory inputs and then feed these results to other central processing neurons in the brain. For instance, consider a person who meets someone they have not seen in a long time. To remember the identity of this person multiple sensory inputs may be processed. Foremost perhaps is the sense of vision whereby one processes what the person looks like. That may not be enough to recognize the person, as the person may have changed over the

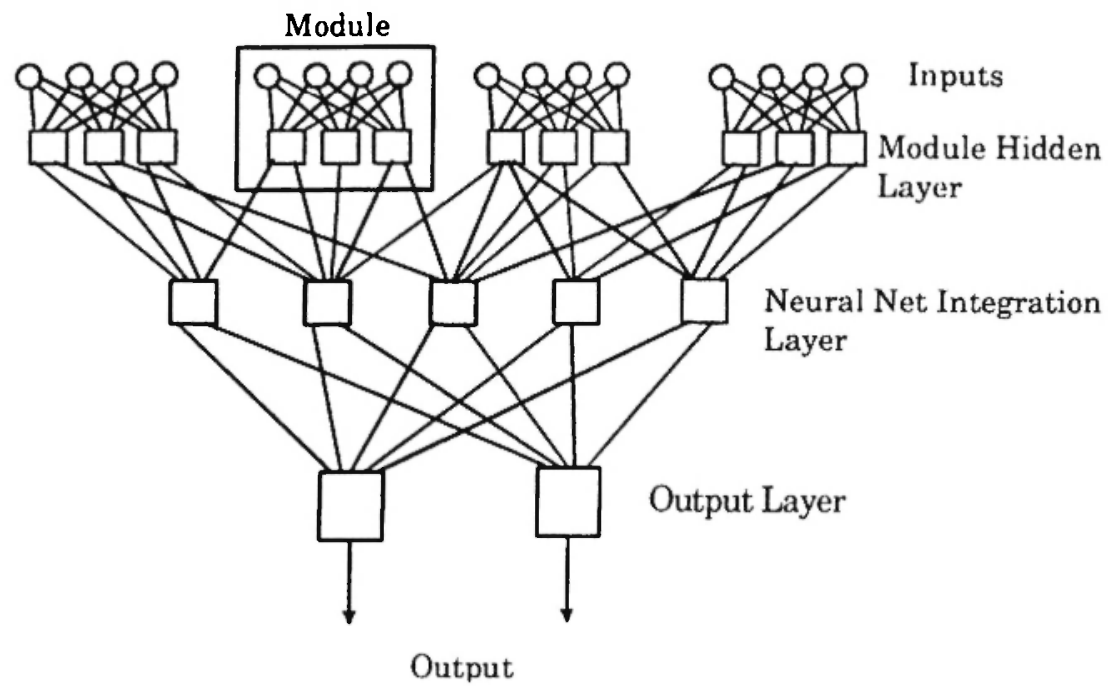

Fig. 3: An Example of a Modular Neural Network: The boxed area highlights one module. The Module Hidden Layer and the Neural Net Integration Layer are not fully connected just for clarity. Normally these two layers would be fully connected Only the input layer to the Module Hidden Layer is modularized. 
course of a number of years. However, their looks coupled with the person's voice, the sensory input from the ears, may be enough to provide an identity. In addition, the olfactory system may also provide more information. In this way our biological system makes many different observations each processed first by some module and then the results sent to be further processed at a central location. Indeed, there may be several layers of processing before a final result is achieved.

In addition to different modules processing the input, the same sensor may process the input in two different ways. While the concept of a modular neural network is based upon biological phenomena, it also makes sense from a purely practical viewpoint. Many real-world problems have a large amount of data points. Using these large number of points as input to a fully connected multilayer perceptron results in a very large number of weights. Just blindly trying to train a network with this approach most often results in poor performance of the network, not to mention long training times because of slow convergence [Haykin, 1994; Rodriguez et al., 1996]. Sometimes there are feature extraction methods, such as those described in section 2, which will reduce the number of data points. However, as was the case in this project, there are times when even then the amount of data is large. Since it is desirable to have the minimum number of weights that will yield good performance, a modular neural network may be a good solution. Each module is effectively able to compress its data and extract subfeatures which then are used as input to a fully connected neural network. Without this modularity the number of weights in the network would be far greater.

\subsection{Neural Network Training}

There are two types of neural network training, supervised and unsupervised [Haykin, 1994; Lippmann, 1987]. Supervised training involves presenting examples to be learned to the network along with the correct output (the teacher) for each example. The calculated outputs are compared to the known values, and the weights are adjusted so that the difference between the outputs and the known values is minimized. The training continues (in theory) until the output for each example is correct. 
Unsupervised training involves presenting examples to the neural network, but without the correct outputs. The weights are adjusted based upon some well-defined algorithm in order to group or cluster like examples together in a consistent manner. Unsupervised training does not require a teacher, and because of this the network's behavior is sometimes called self-organizing. Many scientists believe that the brain operates more like unsupervised training [Wasserman, 1989] and, therefore, in order to attain learning capabilities close to the brain, unsupervised training must be used. In practicality, much success has been achieved with supervised training as well.

This project only considered supervised training. For that reason, the details of different unsupervised training algorithms will be left to the interested reader to research in one of the neural net references [Haykin, 1994; Hecht-Nielsen, 1990; Kung, 1993; Lippmann, 1987; Wasserman, 1989]. While backpropagation is perhaps the most often used supervised training algorithm, because of past performance, ALOPEX was chosen for this system.

\subsection{ALgorithms Of Pattern 'EXtraction - ALOPEX}

The ALOPEX optimization method provides an alternative to the standard gradient descent optimization routines. ALOPEX was originally developed by Tzanakou and Harth in 1973 for receptive field mapping in the visual system of frogs [Tzanakou and Harth,, 1973; Harth and Tzanakou,, 1974]. However, since then it has been used in a wide range of optimization applications [Micheli-Tzanakou, 1995]. Some of these applications include its use with neural networks in the following ways: handwritten digit recognition [Dasey, 1991; Dasey and Micheli-Tzanakou, 1992a], face recognition [Micheli-Tzanakou et al., 1995], analysis of visual-evoked potentials [Dasey and Micheli-Tzanakou, 1992b], analysis of echocardiograms [Micheli-Tzanakou et al., 1993] and speech recognition [Micheli-Tzanakou, 1995]. Other uses of ALOPEX include template matching [Zahner and Micheli-Tzanakou, 1995] in both one and two dimensions. 
In general, optimization algorithms attempt to maximize or minimize some cost function $\mathrm{F}(\mathrm{)}) \mathrm{F}$ is normally dependent on a large number of parameters and, therefore, finding the best solution is difficult. The only way to be certain that a globally optimum solution has been found is to perform an exhaustive search of the parameter space [Zahner and Micheli-Tzanakou, 1995]. However, the parameter space grows exponentially with the number of parameters. If there are a large number of parameters each of which can take on a large number of values, the size of the parameter space is absolutely gigantic. For example, 20 parameters, each of which can have 5 discrete values (a very small parameter space) has $5^{20}=95.4$ trillion possible solutions! To put this in perspective if a computer could perform 1 million evaluations of $F$ per second and it ran 24 hours per day, 7 days per week, it would take just over 3 years to evaluate all possible solutions to this small parameter space. Because of the cost of this exhaustive search, intelligent algorithms that locate near-optimal solutions are acceptable.

ALOPEX performs its optimization based upon both local and global information. If $F\left(x_{1}, x_{2}, x_{3}, \ldots, x_{n}\right)$ is the function to be minimized (also referred to as the cost function or response function), then the ALOPEX algorithm changes all the $\mathrm{x}_{\mathrm{i}} \mathrm{s}$ simultaneously by the formula [Zahner and Micheli-Tzanakou, 1995]:

$$
x_{i}(n)=x_{i}(n-1) \pm \gamma \Delta x_{i}(n) \Delta F(n)+r_{i}(n)
$$

where

$$
\begin{aligned}
& \Delta x_{i}(n)=x_{i}(n-1)-x_{i}(n-2) \\
& \Delta F(n)=F(n-1)-F(n-2)
\end{aligned}
$$

and $r_{1}(n)$ is a random number from a Gaussian distribution with mean 0 and standard deviation $\sigma . \gamma$ is a learning rate parameter used to speed convergence when $\Delta x_{i}$ and $\Delta F$ get small. Note the \pm sign in equation 19. If $F$ is to be minimized then the - sign is used. If $F$ is to be maximized then the + sign is used [Dasey 1991]. 
The cross-correlation term $\Delta \mathrm{x}_{\mathrm{i}} \Delta \mathrm{F}$ is the key to finding the extrema points [Zahner and Micheli-Tzanakou, 1995]. This term provides the direction of movement for the next iteration and is a mixture of local information $\left(\Delta \mathbf{x}_{\mathbf{i}}\right)$ and global information $(\Delta F)$. Consider the situation whereby $F$ is being maximized and at iteration $n, \Delta x_{i}$ was positive while $\Delta F$ was negative. Since $\mathrm{F}$ is being maximized it is desired that $\Delta \mathrm{F}$ be positive. The cross-correlation term in this case is negative which means that $\Delta x_{i}$ will decrease at the next iteration. This is exactly what is desired, since when $\Delta x_{i}$ increased $\Delta F$ decreased, and $\Delta F$ needs to increase which would imply $\Delta x_{1}$ needs to decrease. It is easy to work out the other three combinations and see that in each case the cross-correlation term ensures that the parameters are changed in the direction which helps to maximize $F$. The same scenarios can be worked through to minimize $F$ as well, since with minimizing $F$ the crosscorrelation term is subtracted not added.

The cross-correlation term is not the only factor in updating the parameters. As is well known, one of the problems with backpropagation is that it gets stuck in local extrema. The random noise term $r_{i}$ is added to the change in parameters to give the algorithm a stochastic element. This noise term helps ALOPEX escape local extrema by giving it the possibility of moving in a direction that is not optimal and possibly would be out of the local extrema basin [Dasey and Micheli-Tzanakou, 1992a,b]. However, the value chosen for $\sigma$, the random variable's standard deviation, is crucial. If it is chosen too large then the system will be too random and it may never converge. At the same time, if it is too small the noise term will not be enough to allow the algorithm to break out of local extrema. Additionally, $\sigma$ must be changed as the number of iterations increases [Dasey and MicheliTzanakou, 1992(a,b); Zahner and Micheli-Tzanakou, 1995]. Initially, $\sigma$ should be relatively large in order to not be trapped in local extrema. However, as ALOPEX converges the cross-correlation term becomes smaller. $\sigma$ must follow this trend so that the random noise term does not overpower the cross-correlation term and the algorithm fails to converge.

For ALOPEX to perform the best, $\gamma$ must also be chosen and modified carefully. Initially $\Delta \mathrm{F}$ will be large and $\gamma$ should be small so that the cross- 
correlation term's value does not become too large [Zahner and MicheliTzanakou, 1995]. As the process moves toward convergence $\Delta F$ will become smaller. At this point $\gamma$ needs to increase to compensate for the smaller $\Delta F$ values in the cross-correlation term. This change ensures that the convergence does not become too slow.

Additionally, a bound needs to be placed on the cross-correlation term so that the changes are not too drastic from one iteration to the next [Zahner and Michleli-Tzanakou, 1995]. While fast convergence is desired, steps that are too large in any one direction may move the process away from the desired optimal solution. In ALOPEX drastic changes tend to lead to long periods during which the process oscillates and then fails to converge [Zahner and Micheli-Tzanakou, 1995]. Early in the process larger steps can be tolerated. However, once the algorithm nears convergence smaller steps ensure that the method does not jump out of the attractor basin. For this reason better performance is achieved if this maximum change term is also decreased as the number of iterations increases.

ALOPEX tends to perform better than backpropagation in several ways. First of all, due to its stochastic nature it tends not to get stuck in local extrema. It still may converge to a local extremum, but the tendency is far less than backpropagation. Also, ALOPEX will usually converge much faster than backpropagation, and the difference between its validation performance and its training performance is usually less than backpropagation [Dasey and Micheli-Tzanakou, 1991] (meaning that it remembers more of what it learns). Additionally, since ALOPEX updates all parameters at once, and only once per epoch, it trains faster and is more easily implemented [Zahner and Micheli-Tzanakou, 1995]. However, ALOPEX is somewhat sensitive to the $\sigma, \gamma$, and maximum change values. If these values are too high or too low then ALOPEX's performance may not be satisfactory. While some general guidelines do exist these parameters are, for the most part, problem dependent. 


\section{Classification Results}

As noted in section 3, classification was performed by a modular neural network with 9 total modules. The input to the modules were features extracted by taking the moments of the multiresolution wavelet transform of the digitized mammogram. In addition, the moments and entropy of the original mammogram were used as input as well.

Over 250 different experiments were performed with many different neural network structures and parameters. There are literally thousands of combinations that are possible. Table 1 lists the major variables for the classification process.

The kinds of things that can be changed for that type of variable or some possible values for that variable are listed in parentheses. Each one of the subvariables can take on several values of its own. Table 2 lists the best results achieved for certain configurations of the neural network. All of the results listed in table 2 are for 9 modules, 8 with 29 inputs (28 for the one case where entropy was not included) and 1 with 8 inputs ( 7 for the one case without entropy), with 49 training mammograms and 10 test mammograms. In all cases the ALOPEX parameters were changed by exponential decay for $\sigma$ and the maximum change per epoch, and exponential growth for $\gamma$ with a scaling factor of 2500 epochs. In addition the least squares function was used as the cost function in the ALOPEX algorithm.

\section{Table 1}

Types of Variables in the Classification Process

\begin{tabular}{|c|}
\hline Neural Net Structure (Hidden Nodes, Output Nodess) \\
\hline Presentation of Data (By Component, By Octave, Iinropy) \\
\hline Data Nommalization (In and Scale, tanh, Sigmoidal) \\
\hline AI,OPIXX Parameters ( $\sigma, \gamma$, and Maximum (hange) \\
\hline Change of $A \mathrm{I}, \mathrm{OP}$ : $: X$ Parameters (lexponential, tanh, Sigmoidal) \\
\hline Number of Files for Training and Testing \\
\hline
\end{tabular}


Table 2

Classification Results

\begin{tabular}{|c|c|c|c|c|c|c|c|}
\hline & $\begin{array}{l}\text { Neural Nel } \\
\text { Structure }\end{array}$ & 1):ta Non!l & $\begin{array}{l}\text { Nl.()P:X Parauns } \\
\text { o. } \gamma \text {. Milx (hamLe }\end{array}$ & ling $\%$ & lest $X_{2}$ & $\begin{array}{l}\text { Scnsitivity } \% \\
\text { Specilicity } \% \\
\end{array}$ & 1:pkchs \\
\hline$I$ & $\begin{array}{c}29-3-8-2 \\
8-2 \\
\text { Entropy } \\
\end{array}$ & Double in & $\begin{array}{l}0.120-0.003 \\
0.220-25.00 \\
0.175-0.025\end{array}$ & 93.9 & 100.0 & $\begin{array}{l}I(x) .0 \\
I(x) .0\end{array}$ & $362(x)$ \\
\hline 2 & $\begin{array}{c}29-3-8-2 \\
8-2 \\
\text { Entropy } \\
\end{array}$ & I rouble in & $\begin{array}{l}0.1(x) \cdot 0.00 .3 \\
0.200-25.00 \\
0.170-0.025 \\
\end{array}$ & 93.9 & 90.0 & $\begin{array}{c}75.0 \\
I(x) .0\end{array}$ & $234(x)$ \\
\hline 3 & $\begin{array}{c}29-3-8-2 \\
8-2 \\
\text { Entropy } \\
\end{array}$ & Double ln & $\begin{array}{l}0.150-0.003 \\
0.275-25.00 \\
0.200-0.025 \\
\end{array}$ & 918 & 90.0 & $\begin{array}{c}750 \\
100.0\end{array}$ & $39+(x)$ \\
\hline 4 & $\begin{array}{c}29-3-8-2 \\
8-2 \\
\text { lintropy } \\
\end{array}$ & I rouble In & $\begin{array}{l}0.150-0.003 \\
0.270-25 .(x) \\
0.225-0.025 \\
\end{array}$ & 91.8 & 80.0 & $\begin{array}{l}75.0 \\
83.3\end{array}$ & $154(x)$ \\
\hline 5 & $\begin{array}{c}29 \cdot 3-9-2 \\
8-2 \\
\text { Entrony }\end{array}$ & Touble in & $\begin{array}{l}0.100-0.003 \\
0.200-25.00 \\
0.150-0.025 \\
\end{array}$ & 91.8 & 80.0 & $\begin{array}{l}75.0 \\
83.3\end{array}$ & $28(x)(x)$ \\
\hline 6 & $\begin{array}{c}29-3-8-2 \\
8-2 \\
\text { Entrony } \\
\end{array}$ & mouble in & $\begin{array}{l}0.250-0.003 \\
0.330-25 .(x) \\
0.400-0.025 \\
\end{array}$ & 89.8 & 80.0 & $\begin{array}{l}50.0 \\
100.0\end{array}$ & 20200 \\
\hline 7 & $\begin{array}{c}29-3-7-2 \\
8-2 \\
\text { Entropy } \\
\end{array}$ & I rouble [n & $\begin{array}{l}0.150-0.003 \\
0.250-25 .(x) \\
0.225-0.025\end{array}$ & 87.8 & 80.0 & $\begin{array}{l}75.0 \\
83.3\end{array}$ & $35410)$ \\
\hline 8 & $\begin{array}{c}28-3-8-2 \\
7-2 \\
\text { No Entrony } \\
\end{array}$ & Double in & $\begin{array}{l}0.120-0.003 \\
0.220-25(x) \\
0.175-0.025 \\
\end{array}$ & 95.9 & 70.0 & $\begin{array}{l}75.0 \\
66.7\end{array}$ & $36(x) 0)$ \\
\hline 9 & $\begin{array}{c}29-3-8-1 \\
8-2 \\
\text { Entropy } \\
\end{array}$ & l)ouble in & $\begin{array}{l}0.120-0.003 \\
0.220-25.00 \\
0.175-0.025 \\
\end{array}$ & 93.9 & 70.0 & $\begin{array}{l}50.0 \\
83.3\end{array}$ & $156(x)$ \\
\hline 10) & $\begin{array}{c}29-3-10-2 \\
8-2 \\
\text { Entropv } \\
\end{array}$ & Pouble In & $\begin{array}{l}0.1(x)-0.003 \\
0.200-25.00 \\
0.150-0.025 \\
\end{array}$ & 89.8 & 70.0 & $\begin{array}{l}75.0 \\
66.7\end{array}$ & 24800 \\
\hline 11 & $\begin{array}{c}29-3-8-2 \\
8-1 \\
\text { Entropy } \\
\end{array}$ & Double in & $\begin{array}{l}0.120-0.003 \\
0.220-25.00 \\
0.175-0.025 \\
\end{array}$ & 79.6 & 60.0 & $\begin{array}{l}50.0 \\
66.7\end{array}$ & $308(0)$ \\
\hline 12 & $\begin{array}{c}29-3-8-2 \\
8-3 \\
\text { Entropy } \\
\end{array}$ & Double In & $\begin{array}{l}0.120-0.003 \\
0.220-25.00 \\
0.175-0.025 \\
\end{array}$ & 79.6 & 60.0 & $\begin{array}{l}50.0 \\
66.7\end{array}$ & $138(x)$ \\
\hline 13 & $\begin{array}{c}29-3-8-2 \\
8-2 \\
\text { Entropy } \\
\end{array}$ & Single lin & $\begin{array}{l}0.120-0.003 \\
0.220-25.00 \\
0.175-0.025 \\
\end{array}$ & 59.2 & 60.0 & $\begin{array}{c}0.0 \\
100.0\end{array}$ & 5400 \\
\hline 14 & $\begin{array}{c}29-4-11-2 \\
8-2 \\
\text { Entropy } \\
\end{array}$ & l)ouble in & $\begin{array}{l}0.120-0.003 \\
0.220-25.00 \\
0.175-0.025 \\
\end{array}$ & $9 \times 11$ & 500 & $\begin{array}{l}25.0 \\
66.7\end{array}$ & 8800 \\
\hline 15 & $\begin{array}{c}29-5-12.2 \\
8-2 \\
\text { Entropy }\end{array}$ & Double in & $\begin{array}{l}0.120-0.003 \\
0.220-25.00 \\
0.175-0.025\end{array}$ & 95.9 & 60.0 & $\begin{array}{l}75.0 \\
5(0.0)\end{array}$ & $92(x)$ \\
\hline
\end{tabular}

The first column of the table gives the configuration of the 8 modules which use the moments of the wavelet coefficients as their input. A 29-3-8-2 configuration means each module had 29 inputs, 3 hidden nodes in the 
module followed by 8 nodes in the neural network integration layer and finally 2 output nodes. On the next line in the first column is the configuration of the module which processes the moments of the original image. A 8-2 means that there are 8 inputs into the module with 2 hidden nodes. The neural network integration layer and output layer are the same as the other modules since all the modules feed into these layers. The third line in the first column of the table indicates whether or not the entropy values were included in each module.

The second column shows how the data were normalized. All of these use some variation of the natural logarithm $(l n)$ and then scale these numbers to lie in the $[-1,1]$ interval. Most of the experiments listed perform this process twice since that method performed much better. This is further discussed in the next section.

The third column lists the initial and final ALOPEX parameters. The top line gives the $\sigma$ values, the next line the $\gamma$ values, and the last line the maximum change values. For the final values these are the values that the parameters would be if the network was allowed to run long enough. The final values were reached at approximately 20,000 epochs for $\sigma$ and the maximum change, and 12,000 epochs for $\gamma$.

Columns four and five give the best training and testing percentages, respectively. These percentages were taken once the network had stabilized. The graph in figure 4 gives an example of the convergence of the network. As it can be seen, at a low number of epochs the network oscillates and is not stable. In the graph the network starts to stabilize at approximately 6000 epochs. This point differs from configuration to configuration but all percentages were taken after that point. Also, these percentages are the ones that were repeated and had consistently occurred over the final 600 epochs or so. This eliminates the single data point that may be an anomaly because of the random nature of the ALOPEX algorithm.

Column six of Table 2 lists the sensitivity and specificity of the system as measured on the test data. Sensitivity is a measure of how well the system detects abnormal mammograms and specificity is gauge of the system' $s$ 


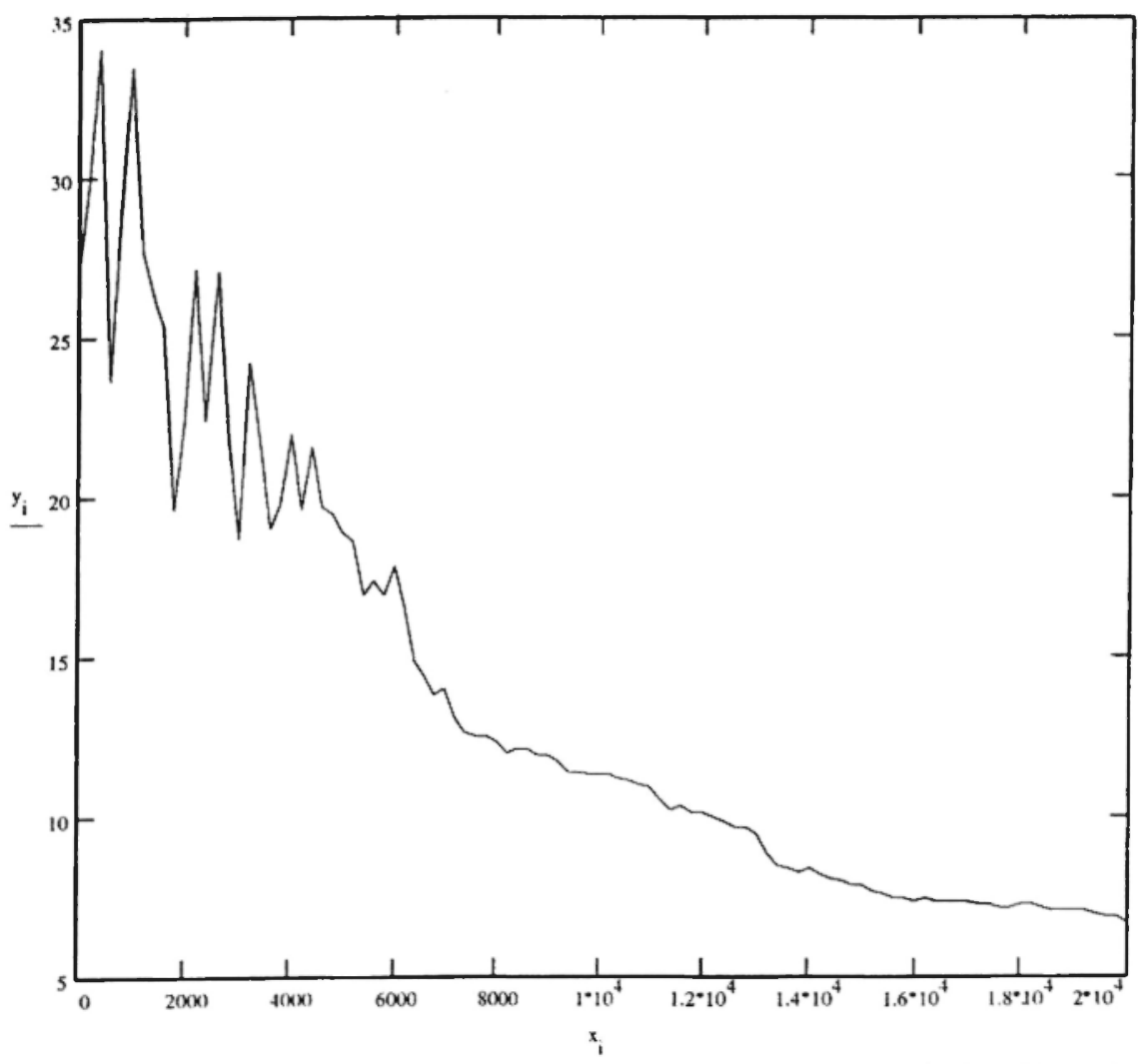

Fig. 4: Graph of the Convergence of One Example of the Neural Network: The number of iterations is on the $\mathrm{x}$-axis and the total error is on the y-axis. The number of iterations only goes to 20,000 , but the graph shows the early initial oscillations.

performance on normal mammograms [Samiy, Douglas, and Barondess 1987]. These quantities are calculated in the following way:

Sensitivity $=\frac{\text { Number of True Positives }}{\text { Number of True Postives }+ \text { Number of False Negatives }}$

Specificity $=\frac{\text { Number of True Negatives }}{\text { Number of True Negatives }+ \text { Number of False Positives }}$ 
where: true positives are those mammograms that the system classifies as abnormal and are truly abnormal, true negatives are those mammograms that the system classifies as normal and are truly normal, false positives are those mammograms that the system classifies as abnormal and are really normal, and false negatives are those mammograms that the system classifies as normal that are really abnormal. The sensitivity and specificity values are important, but because of the small test sample they vary widely for this system. The last column gives the number of epochs required to obtain the performance listed in columns four and five. Most of these experiments were run longer than this number of epochs. However sometimes performance, especially the testing percentage, will decrease if the network trains beyond a certain point. Normally the experiments were run until the performance started to degrade and then they were stopped. Therefore, the epoch numbers represent when the network peaked in its performance. However, many more types of experiments were run than those listed in table 2 . Usually these experiments showed lower performance. The next section will discuss them, since the path that led to the results is often more important than the results themselves.

\subsection{The Process of Obtaining Results}

As in all research projects, the data have to be examined very carefully. First, the range of the moment values is from $-1 \times 10^{41}$ to $1.1 \times 10^{41}$. This is a huge span, but 85 percent of the raw data lie in the $[-1,1]$ interval! In addition, the range of values in one octave is fairly large, but the range in one component is not nearly as large. This makes sense since the moments are based upon the pixel values. Clearly it is easy to see that the residual has the highest concentration of large pixel values and, therefore, these moments will be similar. Following this logic then, it would make sense that the component which contains the vertical edges would have moment values in the same general range as other like components. This is true for the diagonal and horizontal components as well. Also, the moments that were highest in absolute value normally came from the vertical and diagonal 
components. However, no distinguishing characteristics were gleaned from studying the entropy values. There appeared to be no difference between the normal and abnormal cases.

The above insights led to some changes in the neural network structure. However, perhaps more importantly, they led to changes in the way the data were normalized. At the start, the data were normalized by taking the natural logarithm $(I n)$ of all moments and then globally scaling these data onto the $[-1,1]$ interval. What is meant by global scaling is that a max and a min value are determined for the $\ln$ of all the data points and then these values are used to scale the data by the following equation:

$$
\text { scaled value }=\frac{(\text { old value }-\min )}{(\max -\min )} \times 2-1
$$

But since most of the raw data are in the $[-1,1]$ interval and the max and min values are so huge in absolute value, this procedure compacts most of the data into a very small interval. This makes classifying the data difficult since the separation between the data is so small. Therefore, the switch was made to scaling the data by component since the component data is similar in magnitude. In this process, maximum and minimum values for the natural logarithms were calculated for each component and each component was scaled with its corresponding maximum and minimum values. This meant that the first component moments, which are normally very small in magnitude, did not get lost in scaling by huge numbers. These small numbers were now scaled with other small numbers. This meant that now these numbers had some impact upon classification since they were no longer compacted so tightly.

Other normalization methods were tried, such as the hyperbolic tangent and a sigmoidal function, but consistently none worked as well as the $\ln$ and scaling method. In addition, the best approach is to perform this method twice on the data. Potential reasons for this are discussed in the next section.

Given the above changes the next approach was to structure the neural network with 4 modules but each module would process all the moments from one component, not by octave. In addition, the normalization approach 
was changed to scale the In data by component (only once at this time), and the entropy values were added to each module. These experiments started to give some better results. Training percentages near and slightly above 80 percent were achieved. However, the testing percentages were normally between 60 and 70 percent.

Combining the two ideas presented up to this point increased the training percentage. The structure now consisted of 8 modules, 4 of which processed the moments by octave and 4 which processed the moments by component. In addition each module contained the entropy value and the data was normalized using a single $\ln$ and scale method. It was now possible to obtain training percentages approaching, and at times greater than, 90 percent. However, the testing percentage continued to be between 60 and 70 percent.

At this point, analysis of the output from the trials thus far indicated that certain mammograms were consistently mis-classified. From the start, the training had been performed on 52 mammograms, 32 normals and 20 abnormals, and the testing on the remaining 13 in the data base, 8 of which were normal and 5 abnormals. All the "problem" mammograms were normals that would be classified as abnormals. A review of these mammograms showed some interesting things. In one case there was a scar from a previous surgery and the system was picking this up as abnormal. In other cases, metallic markers were put on the breast to mark moles or other exterior-related abnormalities so that the radiologist would know that this spot was not something internal. These markers appear as very bright small dots on the mammogram, and the system was determining that this was abnormal. In yet another case, there were several marks on the mammogram that were made by a person who desired to draw attention to some area. Figure 5 shows an example of a mammogram with metallic markers. After this review, 6 normals were taken out of the data base and only 59 mammograms remained, 34 normals and 25 abnormals.

Further trials with the same structure, but the new data base, now quite often showed training percentages greater than 90 percent. Occasionally the testing percentage would reach 80 percent, but most likely would be 70 percent. At this time it was decided that more information from the original 


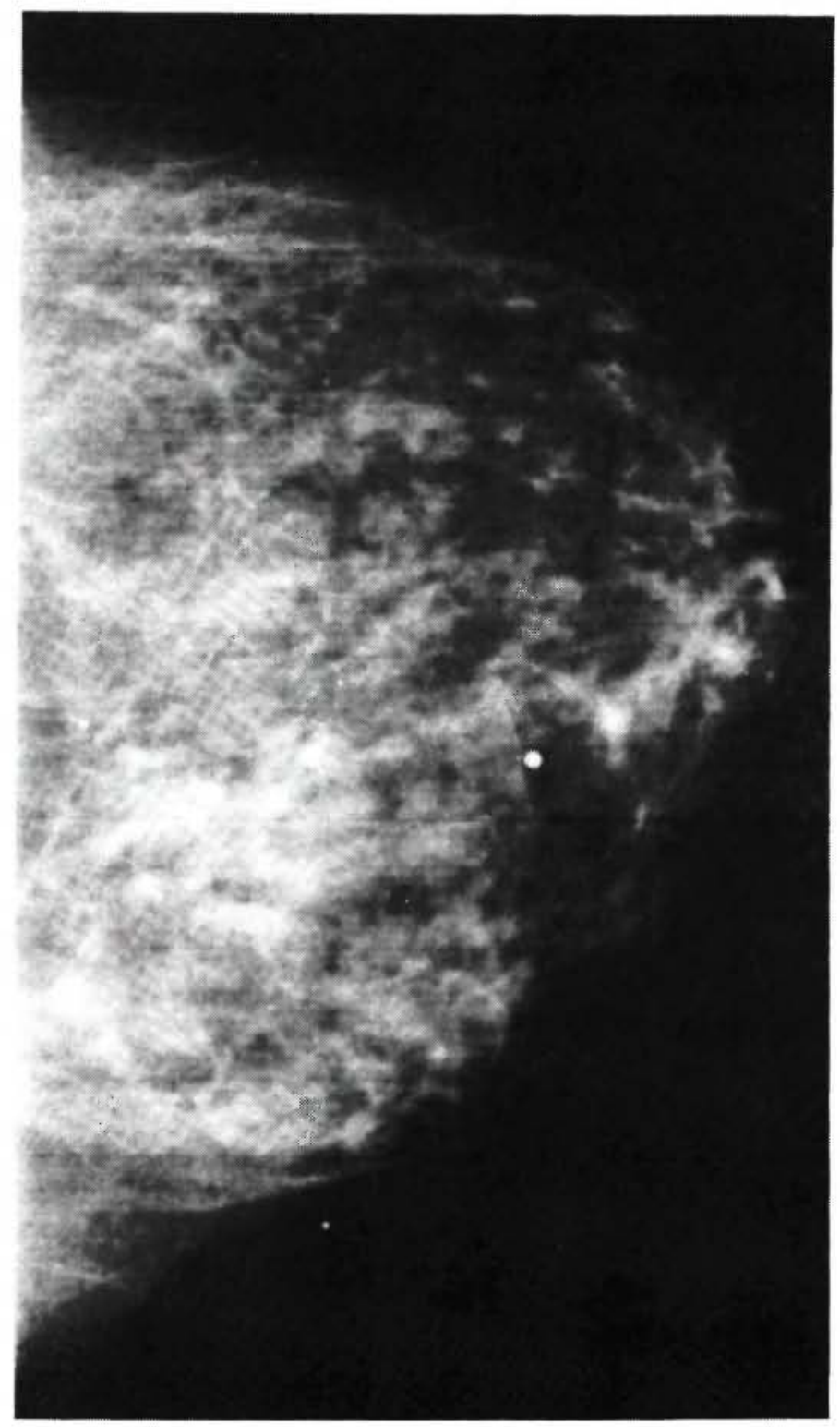

Fig. 5: Example of a Mammogram with Metallic Markers: The two metallic markers are easily seen as bright perfectly round spots on the breast. These spots confused the classification process and this mammogram was consistently misclassified. 
image might aid the classification process. Another module was added that only contained the seven moments from the original image. Experiments with this structure showed a better average performance. However, for the most part the best trials were the same, training greater than 90 percent and testing at 80 percent. In one instance training was performed to 96 percent and testing to 90 percent. The problem was that this result could not be recreated but, in the process of attempting to recreate this result a key concept was discovered.

Our system has the ability to gather the data from a number of files (moment files for each mammogram, entropy files and moment files for the wavelet coefficients) and then save these data to one compact file in order to simplify future experiments on the same data. These data can be saved at any time, even after it has been normalized. To save one processing step the data had been saved after the In and scaling operation. However, in remembering the steps for that experiment it was discovered that the data had again been normalized by performing an additional $\ln$ and scaling operation. While those results with that neural network structure were never consistently recreated this discovery was important. By using this double $\ln$ and scaling method for normalization, the testing percentage was increased on the average. Comparing row 1 and row 13 on Table 2 show an extreme difference between the two normalization methods. Fewer trials ended with a testing percentage of 60 percent and many more achieved 80 percent or higher. However, a reasonably consistent 90 percent result was not yet attainable.

\subsection{ALOPEX Parameters}

Prior to this time the ALOPEX parameters had not been varied much. During the attempt to recreate the results, experiments were done with widely different ALOPEX parameters. Also, at this time experiments were done with changing the updating method for the parameters. Initially it seemed that relatively large starting values for the ALOPEX parameters gave better performance, and, as a result, most trials were done with initial parameters close to: $\sigma=0.25, \gamma=0.35$ and the maximum change per epoch 
being 0.40 . Once the additional module was added, better results were achieved with initial values much less than those listed above such as: $\sigma=$ $0.12, \gamma=0.22$ and the maximum change per epoch equaling 0.175 . A comparison of row 1 with rows 3, 4 and 6 show the difference that the ALOPEX parameters can make and the effect of making the initial values smaller. Row 2 shows that making the values smaller yet does not necessarily increase performance.

While exponential decay for the maximum change and $\sigma$ was always used, the rate of decay was changed. Initially, the experiments were run with a decay rate of 3500 . This means that every 3500 epochs the values are decreased by a factor of $e$, where $e$ is the exponential function. The slower the decay (this translates to a larger decay rate) the more randomness exists in the system for a longer period of time. Also, since the maximum change will be greater for a longer period of time, the less stability the network has in the early training stages. Initially this was thought to be beneficial. However, once the network evolved into its final structure the longer decay rate did not perform as well. After trials with several different rates, a decay rate of 2500 was determined to provide the best results. Figure 6 shows a graph of different decay rates.

The learning rate, $\gamma$, must increase as the network starts to converge. Different growth methods were tried as shown in figure 7. The hyperbolic tangent, with a multiplicative factor to make $\gamma$ greater than 1, would appear to be an ideal growth choice, but it initially grows too slowly. A sigmoidal function was tried, but again the performance of the network was worse with this approach as it too grows too slowly at high epoch numbers. The exponential growth method consistently performed the best, and, while not required, the growth rate was set to be the same as the decay rate for the maximum change and $\sigma$. Trials with other growth rates did not provide any improvement in convergence or performance.

Additionally, the final values for the ALOPEX parameters were varied. At the start of the experiments these values were set to much lower numbers for $\sigma$ and the maximum change, and a much higher number for $\gamma$. The low number for the maximum change led to training that would essentially stop 


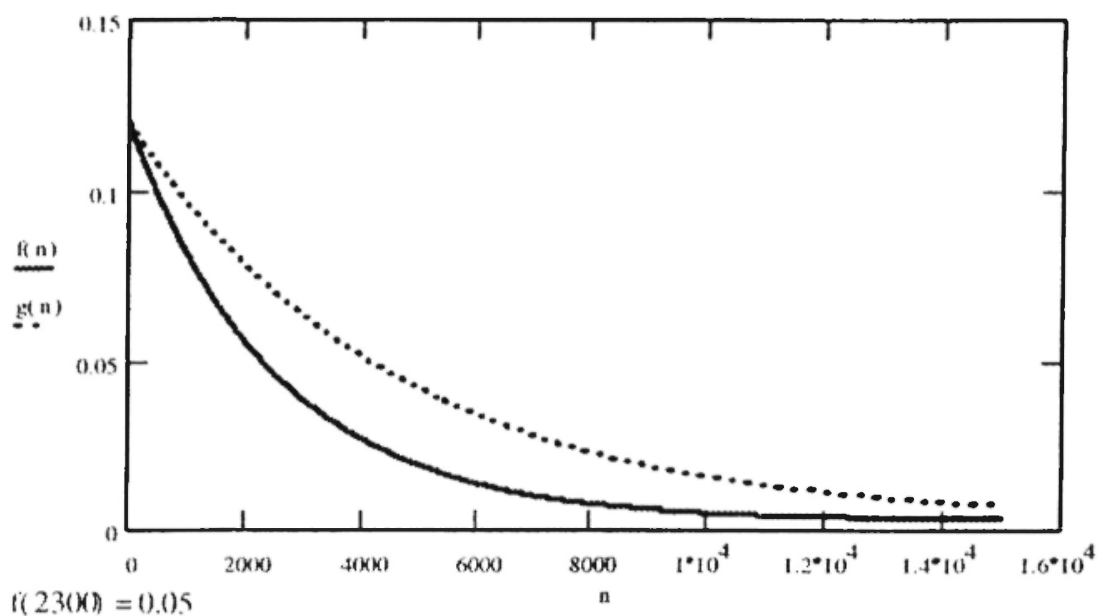

$g(+10)=0.05$

Fig. 6: Graph of Different $\sigma$ Decay Curves: The solid line represents the decay curve with a decay rate of 2500 epochs. The dashed line represents a rate of 4500 epochs. As shown, $\sigma$ is equal to 0.05 at 2300 epochs for the 2500 decay rate and at 4100 epochs for the 4500 decay rate.

once the number of epochs was large enough so that the final value was reached. Furthermore, the lower ending number for sigma virtually eliminated the random component, thereby adding to the slow convergence. Once these numbers were raised, the high number for $\gamma$ caused the system to have difficulty stabilizing in the midtraining stages. Therefore the maximum $\gamma$ value was lowered. The final values were not extensively varied, but the ones given in table 2 seem to perform adequately.

During all the experimentation with the ALOPEX parameters the entropy value was added as an input to the ninth module which processes the moments of the original image. This seemed to be the final change needed and led to the best results of 93.9 percent training and 100 percent testing. While no discriminating value can be seen by examining the entropy value, that in conjunction with the moments definitely appears to provide 


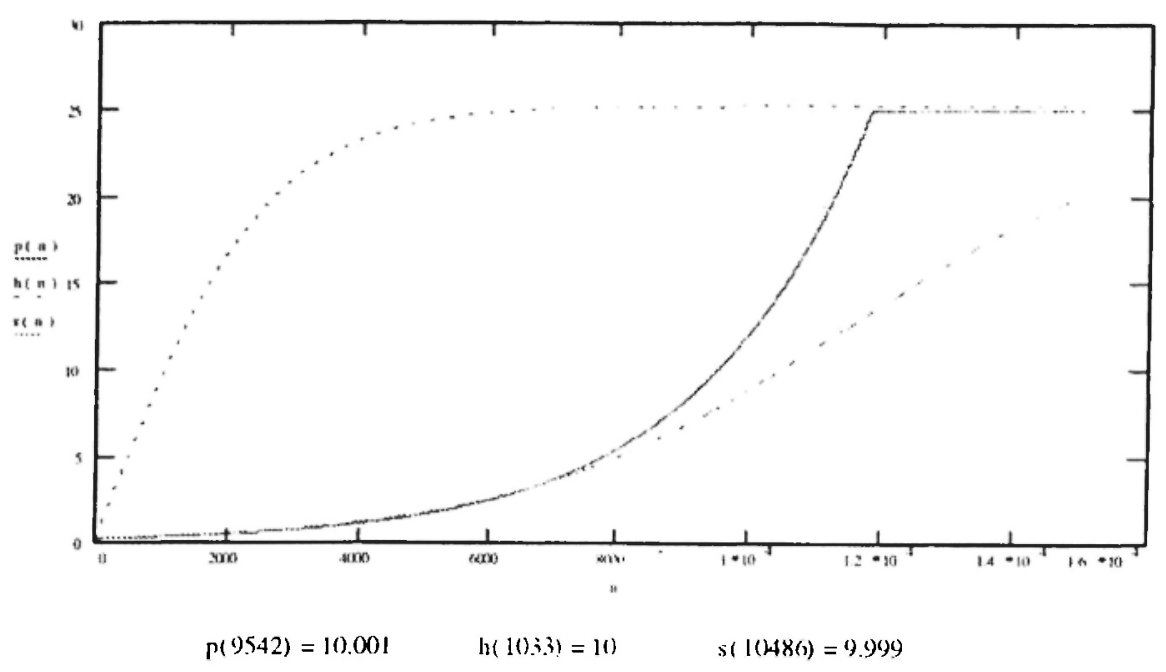

Fig. 7: Graph of Different Growth Curves for $\gamma$ : This graph shows the difference in possible growth curves for $\gamma$. The dotted line is the hyperbolic tangent function multiplied by the maximum value for $\gamma$ which is set at 25 . The solid line is the exponential growth curve that was actually used. When $\gamma$ reaches 25 it no longer increases. The dashed line is the sigmoidal-like function multiplied by 25 and translated so that when $\mathrm{n}=0$ it is the initial value of $\gamma$. Note the different number of iterations needed for each curve to achieve the value of 10 .

good discrimination. Moreover, using these inputs the neural network structure was varied. Comparing rows 1,2 and 3 with rows 5, 7 and 10 of table 2, it is evident that the structure with 3 hidden nodes per module (except for the ninth module with only 2 hidden nodes), 8 integrating neural network nodes, and 2 output nodes gives the best performance.

Additionally, to satisfy curiosity, experiments were performed using only the moments of the original image and the entropy. While only seven different configurations were run, the best result was with 5 hidden nodes in the module feeding to 4 neural net nodes and then to 2 outputs. This gave 75.5 percent training and 50.0 percent testing. Experiments were also 
performed using a fully connected neural network. The configuration contained the same number of nodes as the best modular network, giving 240 input nodes, 26 nodes in the first hidden layer, 8 nodes in the second hidden layer, and 2 output nodes. This configuration usually did not converge. When it did converge the outputs were all zeroes giving $\mathbf{5 7 . 2}$ percent training and 60.0 percent testing.

The time to perform one experiment varies with the structure, the computer platform used, and the number of epochs that are required, most of these trials took between 90 and 120 minutes. While it may be possible to use other structures and change the parameters such that equally good results are achieved, this system appears to perform best with the configuration given above. The next section will explain why the system responds as it does to changes in configuration.

\section{Discussion}

There are three areas of the results presented in the last section that require further explanation. They are: the changes in results related to the neural network structure, the changes related to the ALOPEX parameters, and the performance related to the data normalization techniques. Each of these will be discussed in detail. However, a general point that needs to be made is that ALOPEX is a stochastic process that relies upon random numbers. In addition to that, the neural network weights are initialized to random numbers. Because of this, recreating exact results is very difficult. This means that comparing the effects of parameter and neural network structure changes is also a difficult task. Most often, several trials are run with a certain configuration to get a general sense of how well that configuration performs. Therefore, the conclusions reached are based upon general trends. Because of the randomness involved it is certainly possible that one very unique trial in one configuration may be better than a second configuration, even though in general the second configuration will give better results. 


\subsection{Generalization}

Table 2 shows that in 12 of the 15 cases presented the training percentages are at least acceptable. In many cases they are very good. However, really only the first three cases show acceptable testing or validation percentages. The testing percentage tells how well the network can generalize. By generalization we mean how well the network performs on cases it has not seen but that are similar to the training data. Humans are particularly good at this. For example, once children are told what an automobile is, they can correctly identify another automobile even though it may be a different color, shape, or size. Not only that, they could be shown a picture of a boat and they will normally be able to say that it is not an automobile. The goal is to configure the neural network so it can perform as well.

Table 3

Comparison of ALOPEX Parameters

\begin{tabular}{|c|c|c|c|}
\hline & Row I Valuc & Row 4 Valuc & $\%$ (hanlec \\
\hline$\sigma$ & 0.120 & 0.150 & +25 \\
\hline$\gamma$ & 0.220 & 0.270 & +23 \\
\hline Max Change & 0.175 & 0.225 & +29 \\
\hline
\end{tabular}

If a neural network could not generalize it would be essentially useless. A system that only recognizes data that it has already seen could be replaced by a simple look-up table [Wasserman, 1993]. Generalization depends on the size of the training set and the configuration of the network [Haykin, 1994]. The generalization problem is closely related to the problem of polynomial interpolation [Wasserman, 1993]. Since the interpolation problem has been well studied it will be used as an example to illustrate some of the ideas of generalization. 
With enough independent variables any number of nonconflicting ${ }^{2}$ data points can be exactly fitted by an interpolating polynomial. However, oftentimes the generalization performance suffers. The same behavior is exhibited in neural networks.

As shown in Table 2 this system exhibits the classic generalization behavior. In a neural network the weights are the independent variables. There is a direct correlation from this application to the interpolation problem. In this problem a fixed number of data points is used for training and the rest for testing. Then a neural network structure is trained to fit those points. Neural networks with more nodes and, therefore, more weights tend to overfit the data. The ones with too few nodes and weights do not learn the data well enough. The network of the best size is the one that provides the best performance in terms of training and generalization.

In order to see the trends, experiments with similar ALOPEX parameters must be compared. The best comparison is between row 1 in table 2 and rows 14 and 15. In this case we see the classic overfitting situation. The neural networks in rows 14 and 15 have more weights corresponding to more independent variables and have a better training percentage with a much lower testing percentage. Additionally, they converge in less epochs. This is a clear case of overfitting the data. The same situation occurs when rows 1 and 2 are compared with rows 5 and 10, but it is not as obvious from Table 2. Since Table 2 reports the best results, there are times when the training percentage is less than the best training percentage in order to report a better testing percentage. Both rows 5 and 10 trained to a higher percentage, but then the testing percentage would be around 60 percent. Furthermore, row 7 shows that the best performance by a network with less weights is not as good as equivalent networks, such as rows 1,2 , and 3 , with a greater number of free parameters.

As stated above the generalization performance is also dependent upon the size of the training set [Wasserman, 1993]. Therefore, the conclusion is

${ }^{2}$ By nonconflicting, it is meant that for any given input there is only one output. For example, if the input was $(1,2,3)$ and the output was $(6,3)$ every time $(1,2,3)$ was input, $(6,3)$ would be the result. 
that there is an optimum size of the training set to maximize generalization performance.

Indeed, much research was done on this problem in the late 1980s and early 1990s [Baum and Haussler, 1989; Cohn and Tesauro, 1989; Mehrota et al., 1991]. Virtually all of this research was done on standard feedforward neural networks using backpropagation as a training mechanism. This makes the application of these results to this system difficult at best. However, if the modules of the neural network are considered to be just compressing the input features, then the classification process could be viewed as a standard feed-forward neural network with one hidden layer. This would mean that the network structure would be 26 input nodes, 8 hidden nodes and 2 output nodes, all fully connected. This gives a total of 224 weights plus a bias term at each node for a total of 260 free parameters. Wasserman [Wasserman, 1993] points out that if the neural network is viewed as a learning system, then the Vapnik-Chervonenkis [Vapnik and Chervonenkis, 1971] or VC dimension can perhaps be used to estimate the number of training examples required for good generalization. However, for a multilayer neural network there is no explicit formula for calculating the VC dimension [Wasserman, 1993]. The VC dimension is related to the number of weights in the neural network [Baum and Haussler, 1989], and this number is often used as an estimate of the VC dimension. Wasserman [Wasserman, 1993] gives a lower bound on the number of examples needed by noting that for certain classes of problems the generalization error will be greater than or equal to $O(d / m)$, where $O($.$) means "on the order of," d$ is the $\mathrm{VC}$ dimension, and $\mathrm{m}$ is the number of training examples. Substituting the numbers from this system if a generalization error of 10 percent is desired, then:

$$
0.1 \geq \mathrm{O}(260 / \mathrm{m})
$$

or

$\mathrm{m} \geq 2600$ 
Obviously, the system described in this paper achieved generalizations of 90 percent and better with far fewer training examples. This shows that these bounds are very conservative in practice.

This observed contradiction between the theoretical lower bounds and the actual behavior of the neural network in practice has been a point of contention between theorists and practitioners [Wasserman, 1993]. There may be many ways to explain the difference; one being that many times the simplifications that must be made to calculate the bounds on the number of training examples results in the bounds not reflecting reality [Mehrota et al., 1991]. In the case of our system, the answer may lie in the fact that the studies were done on systems much different than this with a different learning algorithm. However, another very plausible reason is that the training examples for this system may contain a high percentage of "boundary samples" [Ahmad and Tesauro, 1989; Mehrota et al., 1991]. Boundary samples, or border patterns in a two-class problem, are examples which lie very close to the boundary between the two classes of data. Ahmad and Tesauro showed a marked increase in generalization performance when a large percentage of boundary samples are chosen to be in the training set. In addition, with a high enough number of boundary samples the total number of examples needed to provide good generalization decreases. In the mammography application it is difficult to determine which examples are boundary samples (which is why most theoretical work is done on the XOR problem or the majority problem). However, given the above discussion it would appear that the training set contains a high number of boundary samples.

As previously stated, the generalization performance is dependent on the size of the training set and the structure of the neural network. Given this, there are then two ways to attack the generalization problem. First, one could set the size of the network and then collect enough data to obtain the performance desired. Or, the data could be collected and then the network sized to provide the best results given the data. Both methods have been approached in the above discussion. However, in practicality the second method, which is how this system evolved, is usually chosen. In many 
situations it would be impossible or very expensive to collect more data. For this reason most researchers will change the network structure to accommodate the data.

Section 4 discussed the resulting data normalization method that this system uses. However, it was rather surprising that the In and scaling approach done twice performed that much better than just a single $\ln$ and scale. The answer behind this lies in an analysis of the data.

As section 4 listed, the moment values lie between $-1 \times 10^{41}$ and $1 \times$ $10^{41}$, with the minimum in absolute value being $1 \times 10^{-39}$. Once the $\ln$ is taken the values range from approximately -90 to 94 . When these values are scaled onto the $[-1,1]$ interval, the values from -90 to 0 fall into $[-1,-.02]$, which is almost half the interval. These values of natural logarithms from 90 to 0 were the original data values that were in the $[-1,1]$ interval. That is where 85 percent of the original moment data lie. Therefore, the majority of the data now occupies half the interval instead of $\frac{1}{10^{41}}$ th of the interval that it did before. This results in the data becoming much more separable. To perform this operation again just further separates the data. Figure 8 shows this graphically. It is easy to see that the solid line, which is the $\ln (|x|)$, provides a good separation on the interval $[-1,1]$. However, the separation is even greater with the $\ln (|\ln (|x|)|)$, as that function has a greater range. Figure 9 shows the same thing, only the results are now scaled to lie on the $[-1,1]$ interval to exactly match the normalization of the data. While the same result can be seen, it is clearer in Figure 8.

This ability to allow the majority of the data to be more influential obviously increased the performance of the network. This says that the discriminating value came from this part of the data and not the few data points that were large in magnitude.

\subsection{ALOPEX Parameters}

Table 2 shows that the same neural network structure with different ALOPEX parameters will produce different results. While this is not too shocking given that the ALOPEX parameters control the amount of 


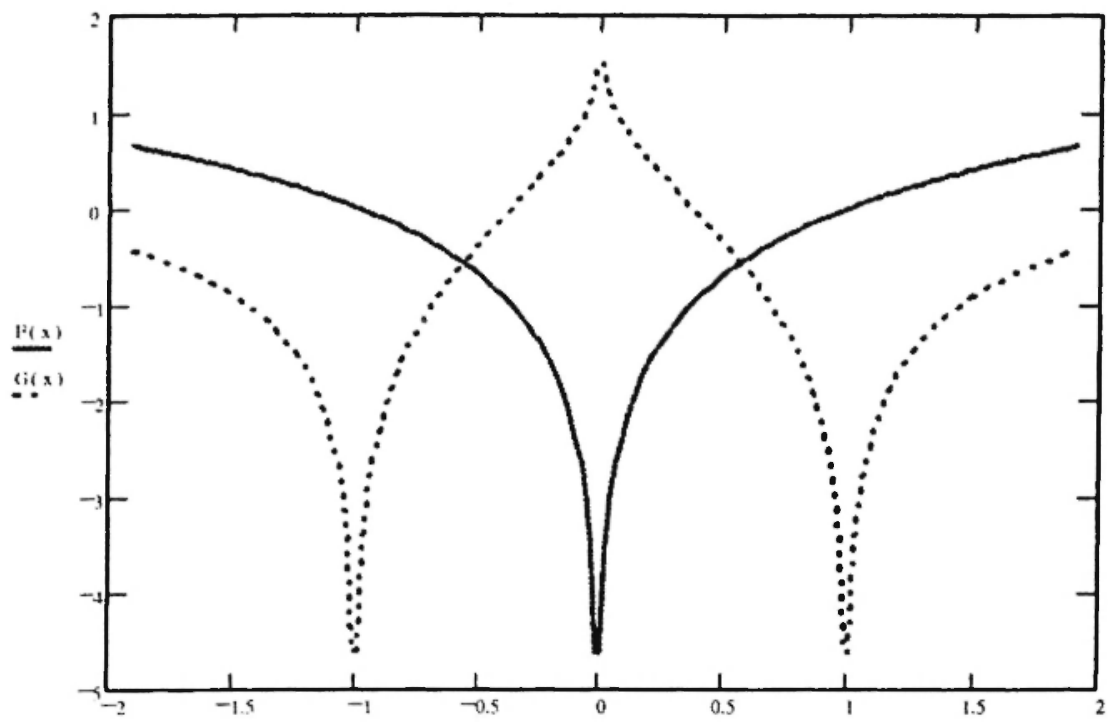

Fig. 8: Graph of $\ln (|x|)$ and $\ln (|\ln (|x|)|)$ : The solid line shows the graph of $\ln (|x|)$ and the dotted line the graph of $\ln (|\ln (|x|)|)$. The interval between -2 and 2 is shown so that the effect on the $[-1,1]$ interval can be more clearly seen.

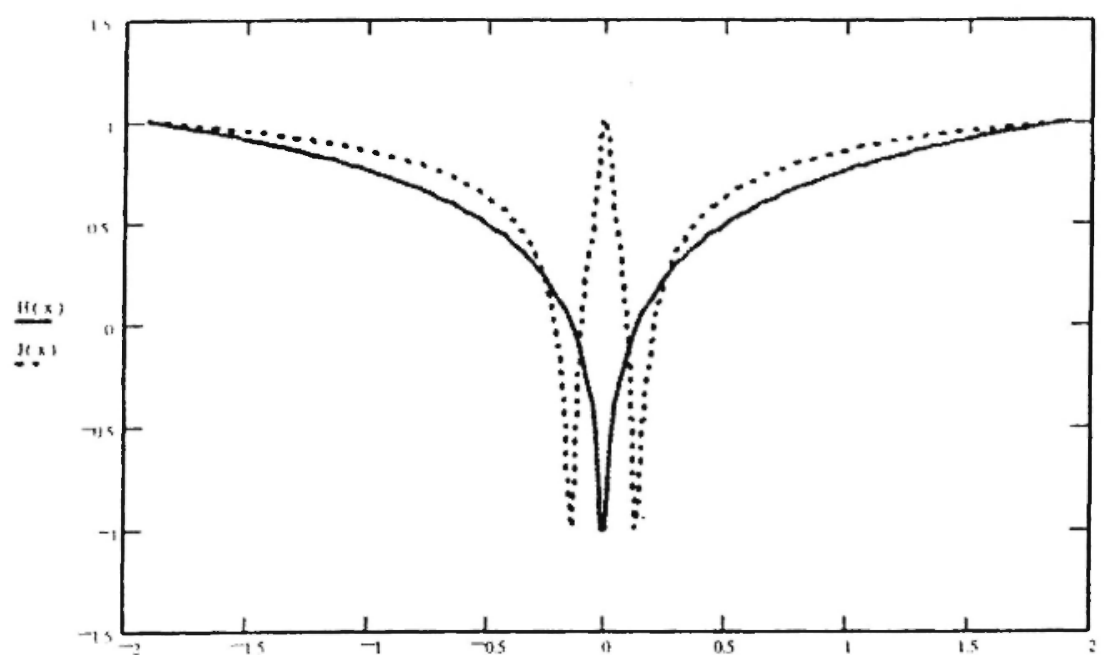

Fig. 9: Graph of $\ln (|x|)$ and $\ln (|\ln (|x|)|)$ with Scaling: This graph shows the same functions as Fig. 8, except that the result of each function is now scaled to lie on the $[-1,1]$ interval as was the input data. 
randomness in the system, for some cases it seems that the parameters are too sensitive to change. For example, comparing row 1 with row 2 shows that a very small change in the ALOPEX parameters led to a drop in the generalization performance. Rows 1 and 4 show an even greater performance drop for a slightly greater change in parameters. This seems to be an undesirable trait. This situation warrants a closer look. Table 3 compares the initial ALOPEX parameters from row 1 and row 4.

When viewed in the perspective of Table 3 things do not seem so out of the ordinary. One would expect that a 25 percent change in parameter values would give a change in performance. Additionally, Table 4 compares rows 1 and 2 which had a small change in performance. Table 4 shows that a smaller percent change in parameters led to this smaller change in performance. This is what would be expected and desired.

Table 4

Comparison of Additional ALOPEX Parameters

\begin{tabular}{|c|c|c|c|}
\hline \multicolumn{1}{|c|}{ Row 1 Value } & Row 2 Value & \%: (hange \\
\hline$\sigma$ & 0.120 & 0.100 & -17 \\
\hline$\gamma$ & 0.220 & 0.200 & -10 \\
\hline Max Change & 0.175 & 0.170 & -3 \\
\hline
\end{tabular}

The system was most sensitive to the starting point of the weights which are randomly set. This behavior is tied to the topography of the error surface, which depends upon the cost or error function used and the number of training examples. While smaller networks have advantages in generalization and training times, as previously discussed, they tend to produce more rugged error surfaces with fewer good solutions [Shang and Wah, 1996]. In this case global minimization methods or methods that use at least some global information tend to perform much better than local minimization methods such as backpropagation [Shang and Wah, 1996]. ALOPEX uses both global and local information and, as such, it is a good choice in this instance. 
The behavior described above is exactly what was observed in this system. There were few good solutions and the ones that existed were difficult to find. To correct this problem additional training examples would need to be used. However, as explained earlier in this section, additional training examples may lead to a larger network required in order to learn the increased training set size. This, in turn, means longer training times. Still, it may be possible to add training examples and smooth out the error surface to alleviate some of the problem and not increase the network size. This is one of the issues for future work.

\section{Conclusions}

We have described a system which reads a digitized mammogram and classifies it as normal or abnormal. The results presented, even though they were generated on a small set of data, show great promise for a system of this type to be used in a clinical setting. With the maturation of digital mammography and the increase in processor speed of readily available and affordable personal computers, this system could easily be fielded on a mobile mammography van. Additional work on a larger data base is ongoing.

\section{Acknowledgments}

The authors would like to thank the United States Air Force and the Rutgers Univ. Research Council for funding support. The mammograms were provided by the New Brunswick Radiology Group (Dr. Barry Zicherman). Our thanks are also extended to Dr. David August of the New Jersey Cancer Institute, for his insightful comments. 


\section{Bibliography}

Ahmad S., and Tesauro G., "Scaling and Generalization in Neural Networks: A Case Study," Advances in Neural Information Processing Systems I, D. Touretzky, ed., Morgan Kaufmann Publishers, Inc., 1989, pp. 160-168.

Antonini M., Barlaud M., Mathieu P., and Daubechies I., "Image Coding Using Wavelet Transform," IEEE Trans on Image Proc, vol. 1, no. 2, Apr 1992, pp. 205-220.

Bankman I., Christens-Barry W., Kim D., Weinberg I., Gatewood O., and Brody W., "Automated Recognition of Microcalcification Clusters in Mammograms," SPIE vol. 1905-Biomedical Image Processing and Biomedical Visualization, [lyear]], pp. 731-738.

Baum E., and Haussler D., "What Size Net Gives Valid Generalization?" Advances in Neural Information Processing Systems I, D. Touretzky, ed., Morgan Kaufmann Publishers, Inc., 1989, pp. 81-90.

Burt P., and Adelson E., "The Laplacian Pyramid as a Compact Image Code," IEEE Trans on Communications, vol. 31, April 1983, pp. 532540.

Cody M., "The Fast Wavelet Transform," Dr. Dobb's Journal, April 1992, pp. 16-28.

Cohn D., and Tesauro G., "Can Neural Networks Do Better than the Vapnik-Chervonenkis Bounds?" Advances in Neural Information Processing Systems 3, D. Touretzky, ed., Morgan Kaufmann Publishers, Inc., 1989, pp. 911-917.

Cowley G. and Ramo J., "Sharper Focus on the Breast," Newsweek, May 10, 1993, pg. 64.

Dasey T. J. and Micheli-Tzanakou E., "A Pattern Recognition Application of the ALOPEX Process with Hexagonal Arrays," IJCNN-89 Vol. II, 119-125 (1989).

Dasey T. J., "Unsupervised Global Optimization Applied to the Classification of Handwritten Digits and Visual Evoked Potentials," Ph. D. Thesis, Rutgers University, 1991. 
Dasey T.J. and Micheli-Tzanakou E., "The Unsupervised Alternative to Pattern Recognition, I.: Classification of Handwritten Digits," Proc. 3rd. Workshop on Neural Networks, Auburn, Alabama pp. 328-333 (1992a). Dasey T.J. and Micheli-Tzanakou E., "The Unsupervised Alternative to Pattern Recognition, II.: Detection of Multiple Sclerosis with the Visual Evoked Potential," Proc. 3rd Workshop on Neural Networks, Auburn, Alabama pp.334-339 (1992b).

Daubechies I., Ten Lectures on Wavelets, SIAM, Philadelphia, PA, 1992.

Davis F., "How to Get the Best Mammogram," Working Woman, Oct 1994, pp. 38-40.

Dhawan A., Buelloni G., and Gordon R, "Enhancement of Mammographic Features by Optimal Adaptive Neighborhood Image Processing," IEEE Trans on Med Imaging, vol. M1-5, no. 1, Mar 1986, pp. 8-15.

Dhawan A. and Le Royer E., "Mammographic Feature Enhancement by Computerized Image Processing," Computer Methods and Programs in BioMed, vol. 27 (1988), pp. 23-35.

Dhawan A., Chitre Y., and Moskowitz M., “Artificial Neural Network Based Classification of Mammographic Microcalcifications Using Image Structure Features," SPIE vol. 1905-Biomedical Image Processing and Biomedical Visualization, 1993, pp. 820-831.

Dodd, Gerald D., MD, "Mammography: State of the Art," Cancer, vol. 53 (1984), pp. 652-657.

D'Orsi C., and Kopans D., "Mammographic Feature Analysis," Seminars in Roentgenology, vol XXVIII, no 3 (July 1993), pp. 204-230.

Elmore J., Wells C., Lee C., Howard D., and Feinstein A., "Variability in Radiologists' Interpretations of Mammograms," New 'Engl Jrnl of Med. vol. 331, no. 22 (1994), pp. 1493-1499.

Feltham R, and Xing G., "Pyramidal Neural Networking for Mammogram Tumor Pattern Recognition," Proc of the IEEE Int Conf on Neural Networks 1994, pp. 3546-3551.

Floyd C., Lo J., Yun A., Sullivan D., and Kornguth P., "Prediction of Breast Cancer Malignancy Using an Artificial Neural Network," Cancer, vol. 74, no. 11, Dec 1994, pp. 2944-2948. 
Friend T., "New Computers can Interpret Mammograms," USA Today, Mar 30, 1995, sect D, pg. 1.

Giger M, Nishikawa R, Doi K., Yin F., Vyborny C., Schmidt R, Metz C., Wu Y., MacMahon H., and Yoshimura H., "Development of a 'smart' Workstation for use in Mammography," SPIE vol 1445 - Image Processing (1991), pp. 101-103.

Gray R., Olshen R., Ikeda D., Cosman P., Perlmutter S., Nash C., and Perlmutter K., "Evaluating Quality and Utility in Digital Mammography," Proc of IEEE Int Conf on Image Proc, 1995, pp. 5-8.

Gold R., "The Evolution of Mammography," Radiologic Clinics of North America, vol 30, no 1, Jan 1992, pp. 1-19.

Goldberg M., Pivovarov M., Mayo-Smith W., Bhalla M., Blickman J., Bramson R., Boland G., Llewellyn H., and Halpren E., "Application of Wavelet Compression to Digitized Radiographs," $A J R$, vol 163, August 1994, pp.463-468.

Gonzalez R., and Wintz P., Digital Image Processing, Addison-Wesley Publishing Company, Reading, MA, 1977.

Harth E. and Tzanakou E. (1974). "ALOPEX: A stochastic method for determining visual receptive fields," Vis. Res., 14, 1475-1482.

Haykin S., Neural Networks - A Comprehensive Foundation, Macmillan College Publishing Company, Inc., 1994.

Hecht-Nielsen R, Neurocomputing, Addison-Wesley Publishing Company, Reading MA, 1990.

Held G., Data Compression, John Wiley \& Sons, New York, NY, 1987.

Hlawatsch F., and Boudreaux-Bartels G., "Linear and Quadratic Time-

Frequency Signal Representations," IEEE SP Magazine, April 1992, pp. 21-62.

Hrycej T., Modular Learning in Neural Networks, John Wiley \& Sons, Inc., New York, NY, 1992.

Hu M., "Visual Pattern Recognition by Moment Invariants," IRE Trans on Information Theory, no 8, Feb 1962, pp. 179-187.

Isard H., MD., "Other lmaging Techniques," Cancer, vol. 53 (1984), pp. 658-664. 
Jiang Y., Nishikawa R, Wolverton D., Metz C., Giger M., Schmidt R, Vyborny C., and Doi K., "Malignant and Benign Clustered Microcalcifications: Automated Feature Analysis and Classification," Radiology 1996, vol 198, no 3, pp. 671-678.

Kallergi M., Woods K., Clarke L., Qian W., and Clark R., "Image Segmentation in Digital Mammography: Comparison of Local Thresholding and Region Growing Algorithms," Computerized Medical Imaging and Graphics, vol. 16, no. 5 (1992), pp. 323-331.

Karssemeijer N., "Recognition of Clustered Microcalcifications Using a Random Field Method," SPIE vol. 1905-Biomedical Image Processing and Biomedical Visualization, pp 776-786.

Kim Y., Choi I., Lee I., Yun T., and Park K., "Wavelet Transform Image Compression using Human Visual Characteristics and a Tree Structure with a Height Attribute," Optical Engineering, vol. 35, no. 1, Jan 1996, pp. 204-212.

Kung S., Digital Neural Networks, PTR Prentice Hall, Englewood Cliffs, NJ, 1993.

Laine A., Fan J., and Yang W., "Wavelets for Contrast Enhancement of Digital Mammography," IEEE. Engineering in Medicine and Biology, Sept/Oct 1995, pp. 536-550.

Laine A., Schuler S., Fan J., and Huda W., "Mammographic Feature Enhancement by Multiscale Analysis," IEEE Trans on Med Imaging, vol. 13, no. 4, Dec 1994, pp. 725-740.

Levine M., Vision in Man and Machine, McGraw-Hill Book Company, New York, NY, 1985.

Li H., Kallergi M., Clarke L., Jain V., and Clark R., "Markov Random Field for Tumor Detection in Digital Mammography," IEEE Trans on Med Imaging, vol 14, no 3, Sept 1995, pp. 565-576.

Lippmann R., "An Introduction to Computing with Neural Networks," IEEE. ASSP Magazine, April 1987, pp. 4-22.

Mallat, S., "A Theory for Multiresolution Signal Decomposition: The Wavelet Representation," IEEE Trans on Pattern Analysis and Mach Intel, vol. 11, no. 7, Jüly 1989, pp. 674-693. 
Mehrotra K., Mohan C., and Ranka S., "Bounds on the Number of Samples Needed for Neural Learning," IEEE. Trans on Neural Networks, vol. 2, no. 6, Nov 1991, pp. 548-558.

Micheli-Tzanakou, E., Yi, C., Kostis, W.J., Shindler, D.M. and Kostis, J.B.: "Myocardial Infarctions: Diagnosis and Vital Status Prediction Using Neural Networks", Computers in Cardiology '93, pgs. 229-232 (1993).

Micheli-Tzanakou E., Uyeda E., Ray R., Sharma A., Ramanujan R., and Doug J., "Comparison of Neural Network Algorithms for Face Recognition," Simulation, vol. 64, no. 1, July 1995, pp. 15-27.

Micheli-Tzanakou E., "Neural Networks in Biomedical Signal Processing," The Biomedical Engineering Handbook, J Bronzino, Ed., CRC Press, Inc., 1995 , ch. 60 , pp. 917-932.

Myers L., Rogers S., Kabrisky M., and Burns R., "Image Perception and Enhancement for the Visually Impaired," IEEE Engineering in Medicine and Biology, Sept/Oct 1995, pp. 594-602.

Qian W., Clarke L., Li H., Clark R, and Silbiger M. (b), "Digital Mammography: M-Channel Quadrature Mirror Filters (QMFs) for Microcalcification Extraction," Comp Med Imaging and Graphics, vol. 18, no. 5, Sept/Oct 1994, pp. 301-314.

Qian W., Clarke L., Zheng B., Kallergi M., and Clark R, "Computer Assisted Diagnosis for Digital Mammography," IEEE Engineering in Medicine and Biology, Sept/Oct 1995, pp. 561-569.

Reeves A., Prokop R., Andrews S., and Kuhl, F., "Three-Dimensional Shape Analysis Using Moments and Fourier Descriptors," IEEE Trans on Pattern Analysis and Machine Intelligence, vol 10, no 6, Nov 1988, pp. 937-943.

Richardson W., “Applying Wavelets to Mammograms," IEEE Engineering in Medicine and Biology, Sept/Oct 1995, pp. 551-560.

Rioul O., and Vetterli M., "Wavelets and Signal Processing," IEEE Signal Processing Magazine, Oct 1991, pp. 14-38.

Rodriguez C., Rementeria S., Martin J., Lafuente A., Muguerza J., and Perez J., "A Modular Neural Network Approach to Fault Diagnosis," IEEE Trans on Neural Networks, vol. 7, no. 2, Mar 1996, pp. 326-340. 
Ross S., A First Course in Probability, Macmillan Publishing Co. Inc., New York, NY, 1976.

Samiy, A., Douglas, R., Jr., and Barondess, J., Textbook of Diagnostic Medicine, Lea and Febiger, Philadelphia, PA, 1987

Shang Y., and Wah B., "Global Optimization for Neural Network Training," Computer, vol. 29, no. 3, Mar 96, pp. 45-54.

Shen L., Rangayyan R, and Desautels J., "Application of Shape Analysis to Mammographic Calcifications," IEEE Trans on Med Imaging, vol. 13, no. 2, June 1994, pp. 263-274.

Sheng Y., "Wavelet Transform," in The Transforms and Applications Handbook, A. Poularikas, Ed., CRC Press, Inc., 1996, ch. 10, pp. 747827.

Sickles E., "Breast Calcifications: Mammographic Evaluation," Radiology (160), 1986, pp. 289-293.

Strang G., "Wavelets and Dilation Equations: A Brief Introduction," SLAM, Review, vol. 31, Dec 1989, pp. 614-627.

Strang G., "Wavelets," American Scientist, vol 82, May/June 1994, pp. 250255.

Strickland $R$, and Hahn $H$,, "Wavelet Transforms for Detecting Microcalcifications in Mammograms," IEEE Trans on Med Imaging, vol. 15, no. 2, April 1996, pp.218-229.

Thomas G., and Finney R, Calculus and Analytic Geometry, AddisonWesley Publishing Company, Reading, MA, 1988.

Tzanakou E. and Harth E. (1973). "Determination of visual receptive fields by stochastic methods," Biop'hys. J., 14 (42a).

Vapnik V. N., and Chervonenkis A. Y., "On the Uniform Convergence of Relative Frequencies of Events to Their Probabilities," Theoretical Probability and Its Applications, vol. 17, 1971, pp. 264-280.

Vyborny C., and Giger M., "Computer Vision and Artificial Intelligence in Mammography," AJR, vol 162, Mar 1994, pp. 699-708.

Wasserman P., Advanced Methods in Neural Computing, Van Nostrand Reinhold, New York, NY, 1993. 
Wasserman P., Neural Computing: Theory and Practice, Van Nostrand Reinhold, New York, NY, 1989.

Woods J., and O'Neil S., "Subband Coding of Images," IEEE Trans on Acoustics, Speech, and Signal Processing, vol 34, Oct 1986, pp. 12781288.

Yoshida H., Zhang W., Cai W., Doi K., Nishikawa R, and Giger M., "Optimizing Wavelet Transform Based on Supervised Learning for Detection of Microcalcifications in Digital Mammograms," Proc of IEEE Int Confon Image Proc, 1995, pp. 152-155

Yu F., and Li Y., “Applications of Moment Invariants to Neural Computing for Pattern Recognition," SPIE vol 1297 - Hybrid Image and Signal Processing II, 1990, pp. 307-316.

Zahner D. and Micheli-Tzanakou E., “Artificial Neural Networks: Definitions, Methods, Applications," The Biomedical Engineering Handbook, J Bronzino, Ed., CRC Press, Inc., 1995, ch. 184, pp. 26992715.

Zettler W.: Huffman J., and Linden D., "Application of Compactly Supported Wavelets to Image Compression," SPIE vol 1244, Image Processing Algorithms and Techniques, 1990, pp. 150-160. 
